\title{
Estimate of significant wave height from non- coherent marine radar images by multilayer perceptrons
}

\author{
Raúl Vicen-Bueno ${ }^{*}$, Cristina Lido-Muela and José Carlos Nieto-Borge
}

\begin{abstract}
One of the most relevant parameters to characterize the severity of ocean waves is the significant wave height $\left(H_{s}\right)$. The estimate of $H_{s}$ from remotely sensed data acquired by non-coherent X-band marine radars is a problem not completely solved nowadays. A method commonly used in the literature (standard method) uses the square root of the signal-to-noise ratio (SNR) to linearly estimate $H_{s}$. This method has been widely used during the last decade, but it presents some limitations, especially when swell-dominated sea states are present. To overcome these limitations, a new non-linear method incorporating additional sea state information is proposed in this article. This method is based on artificial neural networks (ANNs), specifically on multilayer perceptrons (MLPs). The information incorporated in the proposed MLP-based method is given by the wave monitoring system (WaMoS II) and concerns not only to the square root of the SNR, as in the standard method, but also to the peak wave length and mean wave period. Results for two different platforms (Ekofisk and FINO 1) placed in different locations of the North Sea are presented to analyze whether the proposed method works regardless of the sea states observed in each location or not. The obtained results empirically demonstrate how the proposed non-linear solution outperforms the standard method regardless of the environmental conditions (platform), maintaining real-time properties.
\end{abstract}

Keywords: significant wave height, marine radar, multilayer perceptrons, neural networks, sea surface, ocean waves

\section{Introduction}

Ocean waves are oscillations of the free sea surface caused by the wind. Under severe meteorological conditions, ocean waves can be dangerous for human marine activities, such as navigation, on- and off-shore management, etc. One of the most important parameters to define the severity of a given ocean wave field is the so-called significant wave height, $H_{s}$, which is usually defined as the average of the one-third largest wave heights of the ocean wave field of study. $H_{s}$ is usually estimated using in-situ sensors, such as buoys, recording time series of wave elevation information. A complementary technique to analyze ocean waves is to use remote sensing imaging methods, such as coherent radars [1-3], or conventional $\mathrm{X}$-band marine radars [4-6], which are non-coherent radars commonly installed in moving vessels, as well as in

\footnotetext{
* Correspondence: raul.vicen@uah.es

Department of Signal Theory and Communications, Superior Polytechnic School, University of Alcalá, Alcalá de Henares, 28805, Madrid, Spain
}

on- and off-shore platforms, or marine traffic control towers. These non-coherent radars image the sea surface at grazing incidence with horizontal polarization. Radar images are caused by the interaction of the electromagnetic fields transmitted by the radar antenna with the sea surface roughness and ripples due to the local wind $[4,7,8]$. This interaction produces a backscatter of the electromagnetic fields, which is commonly known by sailors as sea clutter, and it is an undesirable signal for navigation purposes.

The measurement of ocean waves by non-coherent Xband marine radars is based on the acquisition of temporal sequences of consecutive radar images of the sea surface. Using these data sets, the spatial and temporal variability of the sea surface is analyzed to extract an estimation of the so-called wave spectrum $[4,7,9]$. From this wave spectrum, typical sea state parameters, such us characteristic wave periods, wavelengths and wave propagation directions, can be derived to describe each sea state [6]. One of 
the sea state parameters commonly estimated from the wave spectrum is $H_{s}$. Since non-coherent marine radars are not radiometrically calibrated, $H_{s}$ cannot be directly obtained from the un-scaled (often logarithmically amplified as a function of range) backscatter image values. Due to the un-scaled backscatter values, the wave spectral estimation is not properly scaled, and the total energy of the wave field cannot be directly estimated [9]. It is also possible to estimate $H_{s}$ for the case of non-coherent marine radars by using an extension of the methodology proposed for processing synthetic aperture radar (SAR) images of the sea surface [10]. This methodology is based on the estimation of the signal-to-noise ratio (SNR) [9], where the signal is the spectral energy of the un-scaled wave spectrum, and the noise is related to the spectral energy of the speckle noise in the radar image. Nowadays, this method is used in operational applications, being considered as a standard method for wave analysis using noncoherent X-band marine radar-based sensors in the literature.

The research study presented in this article discusses the limitations of the standard operational method used to estimate $H_{s}$ from marine radar image sequences. From the analysis of these limitations, the incorporation of the SNR is not enough to make accurate $H_{s}$ estimates in some cases (sea states). Therefore, an improved method should incorporate information from this and other sea state parameters derived from the wave spectrum. Since the wave spectrum is mainly a non-linear process relating different wave generation sources (gravity, wind, etc.), the function implemented by the proposed method is expected to be non-linear. Due to the inherent capabilities of artificial neural networks (ANNs) to implement nonlinear functions [11], they are investigated in this article to find a non-linear relationship of $H_{s}$ with SNR and other sea state parameters. In our case of study, the multilayer perceptron (MLP), a kind of feedforward ANN, is considered. This kind of ANN is selected because it has been successfully used in the literature for different purposes when working with non-coherent marine radars. As an example, the capabilities of the MLPs to implement nonlinear functions [11] have been exploited in [12,13] to create non-linear filters able to reduce the sea clutter power. Moreover, due to the reduced computational cost of the MLP once designed, it can be operationally used to report $H_{s}$ estimates in real-time. The performances and operational properties of the proposed MLP-based method to estimate $H_{s}$ is studied in different sea areas, where different sea states are observed. This study will give us information about whether the MLP-based method can be applied in different sea locations or not.

The article is structured in five additional sections. Section 2 deals with the description of the radar-based system used for measuring ocean waves. This section also describes the characteristics of the in-situ measurements used in this research. Section 3 introduces the standard methodology for estimating $H_{s}$ by using conventional non-coherent marine radar systems, including a discussion of its limitations in practical applications. Section 4 describes the new methodology proposed in the article. A description of the way an MLP is used to estimate $H_{s}$, the way it is trained, its computational cost and the way the available data is divided for its design and test is given. Section 5 presents and compares the results achieved by using the standard and proposed MLP-based methodologies when estimating $H_{s}$. Finally, Section 6 summarizes the main conclusions drawn from this research.

\section{Instrumentation and in-situ measurements}

It is known that, under certain conditions, signatures of the sea surface are visible in non-coherent $\mathrm{X}$-band marine radar images $[8,14,15]$. The radar images of the sea surface incorporate the backscatter of the transmitted electromagnetic waves from the short sea surface ripples in the range of the electromagnetic wavelength (e.g., $\approx 3 \mathrm{~cm}$ ) [16]. Thus, swell (e.g., wave fields caused by storms in other geographical locations and propagated to the area of study) and wind sea (e.g., wave fields caused by local storms) become visible as they modulate the backscattered radar signal. Since standard non-coherent X-band marine radar systems allow to scan the sea surface with high temporal and spatial resolutions, they are able to monitor the sea surface in time and space [14]. The combination of the temporal and spatial wave information permits to obtain wave data, such as the wave spectrum, being related to sea state parameters $[4,6,9]$. The use of non-coherent marine radars allows the detection of wave field features from moving ships, as well as from on- and off-shore platforms. As an active microwave remote sensing device, non-coherent $\mathrm{X}$-band marine radars work at grazing incidence and horizontal polarization [15]. Table 1 illustrates the configuration of the conventional X-band marine radar used in our case of study for ocean wave analysis.

\begin{tabular}{ll} 
Table 1 Transmission and reception characteristics of the \\
marine radar used in the experiments \\
\hline Radar system frequency (X-band) & $9.5 \mathrm{GHz}$ \\
Antenna polarization & $\mathrm{H}$ and H \\
Antenna rotation speed & $25 \mathrm{rpm}$ \\
Pulse repetition frequency (PRF) & $1000 \mathrm{~Hz}$ \\
Radar pulse width & $50 \mathrm{~ns}$ \\
Azimuthal range (coverage) & $0-360^{\circ}$ \\
Azimuthal resolution & $0.15^{\circ}$ \\
Distance range (coverage) & $200-4000 \mathrm{~m}$ \\
Range resolution & $7.5 \mathrm{~m}$
\end{tabular}


In our research study, to measure and store the radar data, an A/D converter, such as the WaMoS II [4,5], is used. WaMoS II is an operational wave monitoring system originally developed at the German GKSS Research Center Geesthacht, and is now commercially available from OceanWaveS, GmbH. The marine radar-based system used in our experiments consists of a conventional marine radar, a high speed video digitizer generating marine radar images (WaMoS II hardware) and software incorporated in a standard computer analyzing the images and extracting sea state parameters in real-time (WaMoS II software). A scheme of the used +WaMoS II installation is depicted in Figure 1.

Two different sources of data are used in our experiments. These data were acquired in two different geographical locations of the North Sea, having different oceanographic conditions. The main properties of these platforms and their environmental characteristics are:

- Ekofisk is an oil field complex, property of ConocoPhillips. Ekofisk is located in the Norwegian sector of the North Sea, about $320 \mathrm{~km}$ southwest of Stavanger. Typical sea state conditions in that area present severe wave fields generated by local storms. This geographical location is a wind sea-dominated area. In addition to these wind sea states, swell-dominated wave fields can also be superimposed, having bimodal sea states. These bimodal sea states are highly dangerous for the stability of the marine structures because these structures are attacked by different wave fields propagating with different directions and different wave periods and lengths.

- FINO 1 is an oceanographic research platform, being located in the German basin of the North Sea. This platform is about $45 \mathrm{~km}$ north of the island of
Borkum. FINO 1 is operated by the Federal Maritime and Hydrographic Agency of Germany (BSH) to understand the meteorological conditions in the area to support the deployment of off-shore wind farms. Although the general sea state conditions are similar to the Ekofisk area, the location of FINO 1 presents more influence of swell cases with longer wave lengths than the location of Ekofisk. In general, swell cases present longer wave lengths and wave periods than wind sea cases. In addition, swell wave fields can occur in presence of weak local wind conditions, where the roughness of the sea surface is low, and the backscattering mechanisms are weak.

In both platforms, the radar antenna is located about $30 \mathrm{~m}$ over the mean sea level. In the geographic area of each platform, additional oceanographic and meteorological instrumentation has been deployed. For wave analysis, a DataWell WaveRider buoy is moored in the vicinity of each platform. The buoys and the WaMoS II systems record wave elevation series and radar image sequences, respectively, being synchronized. From these wave elevation records, the buoys provide standard sea state parameters, such as $H_{s}$, wave periods, etc., which complement the sea state information derived from the WaMoS II data. Figure 2 shows the temporal evolution of $H_{s}$ derived from each buoy at their respective locations (e.g., Ekofisk and FINO 1). These temporal evolutions are taken as reference in the experiments described in this article.

\section{Standard method to estimate the significant wave height from radar images of the sea surface}

This section describes the basics of the method commonly used in the literature to estimate $H_{s}$ from marine radar images of the sea surface. This method is called

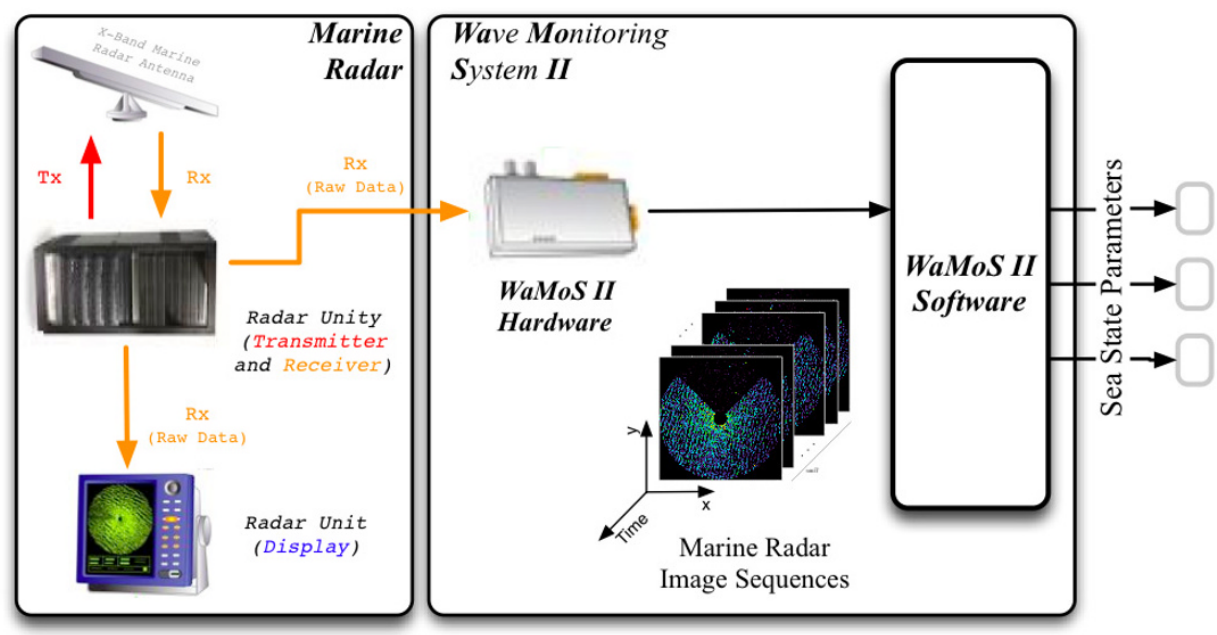

Figure 1 Scheme of the WaMoS II installation used in the experiments. 

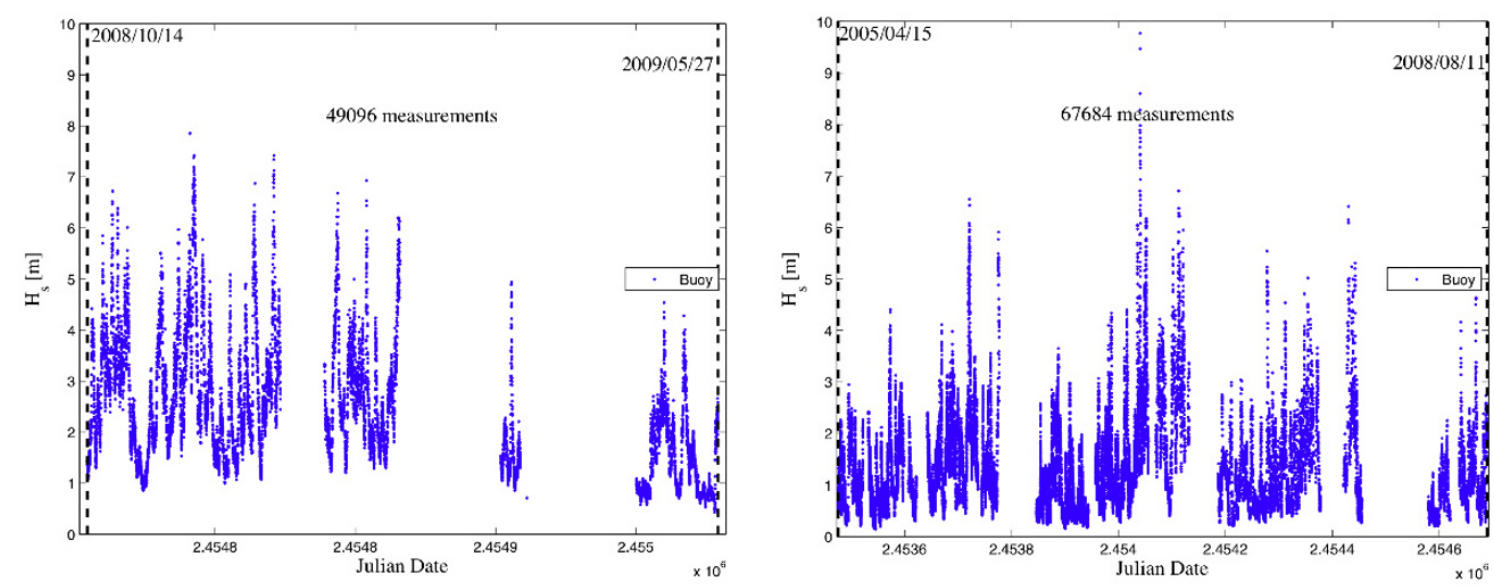

Figure 2 Temporal evolution of $H_{s}$ measured by buoys deployed in the two different radar locations: Ekofisk in Norway, and FINO 1 in Germany. The temporal gaps in the information correspond to periods where the buoys were not operative due to different reasons, such as hardware maintenance, damage of some sensors, etc.

"standard method" in our article. A brief description of the theoretical background of the stochastic wave theory needed to analyze ocean wave fields from marine radar data sets is presented first. The way $H_{s}$ is estimated by the standard method is presented after. The limitations of this method are finally discussed.

\subsection{Spectral representation of ocean waves}

Ocean waves are commonly described by the concept of sea state. A sea state is defined by the area of the ocean and the temporal duration where the wave elevation over the mean sea level can be assumed as a stochastic process with stationary statistical properties in space and time. Under these conditions, the wave elevation $(\eta)$ for a given position of the sea surface $\mathrm{r}=(x, y)$, and for a specific time $t$, is described by the following spectral representation [17]:

$$
\eta(\mathbf{r}, t)=\int_{\Omega_{\mathbf{k}, \omega}} e^{i(\mathbf{k} \cdot \mathbf{r}-\omega t)} d Z(\mathbf{k}, \omega)
$$

where $\omega$ is the angular frequency, being related to the wave period $T$ as $\omega=2 \pi / T ; \mathbf{k}=\left(k_{x}, k_{y}\right)$ is the wave number vector, being defined by a modulus inversely proportional to the wave length $\lambda=2 \pi / k$, and the wave propagation direction is given by $\theta=\tan ^{-1}\left(k_{y} / k_{x}\right)$. The magnitudes $d Z(\mathbf{k}, \omega)$ are the so-called spectral random measures, which are complex amplitudes that determine the energy of each wave component defined by the wave number and frequency $(\mathbf{k}, \omega)$. The integration domain $\Omega$ $\mathbf{k}, \omega=\Omega_{\mathbf{k}} \times \Omega_{\omega}$ is defined by the admissible range of wave lengths and periods that define the ocean waves [18] in opposition to other ocean oscillations, such as tides, planetary waves, etc. In practice, the range of wave lengths and periods that a marine radar can measure is limited by the spatial resolution of the antenna and the antenna rotation period [5]. Equation (1) indicates that the wave elevation can be regarded as a linear superposition of different individual wave components $(\mathbf{k}, \omega)$. It is important to note that the time scale $t$, where both Equation (1) and the temporal sequence of radar images are defined, is shorter than the time scale of the temporal evolution of sea states, which can be seen in the examples shown in Figure 2. Hence, in opposition to $t$, the longer time scale related to the temporal evolution of sea states is denoted in the following as $n$.

The analysis of ocean waves by using non-coherent marine radars is based on the well-known fact that ocean waves are dispersive. Under the frame of the linear hydrodynamic wave theory, the dispersion relation is given by [17]

$$
\omega=\varpi(\mathbf{k})=\sqrt{g k \tanh (k d)}+\mathbf{k} \cdot \mathbf{U},
$$

where $d$ is the water depth and $\mathrm{U}=\left(U_{x}, U_{y}\right)$ is the socalled current of encounter [4].

From Equation (1), the statistical and dynamical properties of the sea state are given by the 3D wave spectrum $F(\mathbf{k}, \omega)[7,17]$, which is commonly defined in the scientific literature as

$$
F(\mathbf{k}, \omega) d k_{x} d k_{y} d \omega=\varepsilon\left[|d Z(\mathbf{k}, \omega)|^{2}\right],
$$

where $\varepsilon$ denotes the expectation operator. The significant wave height, $H_{s}$, can be derived from the wave spectrum as [7]

$$
H_{s}=4 \cdot \sqrt{\int_{\Omega_{\mathbf{k} . \omega}} F(\mathbf{k}, \omega) d k_{x} d k_{y} d \omega .}
$$


In practice, the estimation of $F(\mathbf{k}, \omega)$ takes into account a discretization of the spectral domain $\Omega_{\mathbf{k}, \omega}$ due to the spatial and temporal resolution of the sensor and the size of the total spatial oceanic area of analysis, as well as the temporal duration of the measurement $[4,7]$. For standard ocean and coastal engineering applications, the $3 \mathrm{D}$ spectrum $F(\mathbf{k}, \omega)$ is not used, and there are more practical wave spectral representations defined for lower spectral domain dimensions than $\Omega_{\mathbf{k}, \omega}$. In this study, two different spectral representations are used, being [7]:

- Wave number spectrum:

$$
\bar{F}(\mathbf{k})=\int_{\Omega_{\omega}} F(\mathbf{k}, \omega) d \omega .
$$

From the spectrum $\bar{F}(\mathbf{k})$, an important parameter derived for wave analysis is the wave number $\mathbf{k}_{p}$, where $\bar{F}\left(\mathbf{k}_{p}\right)$ is maximum (e.g., the wave number vector related to the dominant wave component). This parameter is called peak wave number, and it defines a more relevant parameter known as peak wave length $\lambda_{p}=2 \pi / k_{p}$.

- Frequency spectrum:

$$
S(\omega)=\int_{\Omega_{\mathbf{k}}} F(\mathbf{k}, \omega) d k_{x} d k_{y}
$$

The spectrum $S(\omega)$ allows to derive characteristic wave periods of the sea state. One relevant parameter is the mean period $T_{m}$. This parameter considers not only one single wave component, like $\lambda_{p}$, but also a weighted contribution of all the periods related to the different wave components. In ocean engineering, the most usual estimation of $T_{m}$ is known as $T_{m 02}$, which is given by

$$
T_{m}=T_{m 02}=2 \pi \cdot \sqrt{\frac{\int_{\omega} S(\omega) d \omega}{\int_{\Omega_{\omega}} S(\omega) \omega^{2} d \omega} .}
$$

\subsection{Estimation of $H_{s}$ using temporal sequences of radar images}

As mentioned above, the procedure to analyze wave fields is based on the acquisition of temporal sequences of consecutive sea clutter images by using marine radars (see Figure 3). The sampling time of this temporal sequence of images corresponds to the antenna rotation period. The spatial resolutions of each image depends on the azimuthal and range resolutions of the radar.
Applying a 3D Fourier decomposition to the temporal sequence of radar images, the so-called image spectrum $\mathrm{I}(\mathbf{k}, \omega)$ is obtained [7]. This spectrum contains the (k, $\omega)$-components of the temporal sequences of radar images. These components concern to the wave field, as well as other additional contributions, as depicted in Figure 4. These additional contributions are due to the radar imagery mechanisms [7], such as the background noise, $B G N$, caused by the sea surface roughness generated by the local wind $[9,19,20]$, higher harmonics of the dispersion relation (see Equation (2)) due to non-linear radar imagery mechanisms at grazing incidence [19,21], etc. Hence, an inversion modeling technique is applied to the image spectrum $I(\mathbf{k}, \omega)$ to estimate the wave spectrum $F(\mathbf{k}, \omega)$ [7]. The basics of the inversion modeling technique considers the dispersion relation given in Equation (2). Therefore, the estimation of the wave spectrum $\hat{F}(\mathbf{k}, \omega)$ is obtained from the image spectrum $I$ $(\mathbf{k}, \omega)$ by applying a $3 \mathrm{D}$ band-pass filter within the $\Omega_{\mathbf{k}, \omega}$ domain $[4,7]$, which considers only those $(\mathbf{k}, \omega)$-components that holds the dispersion relation $\omega=\varpi(\mathbf{k})$ given by Equation (2), i.e.,

$$
\hat{F}(\mathbf{k}, \omega)=k^{\beta} \cdot \int_{\Omega_{\mathbf{k}, \omega}} I(\mathbf{k}, \omega) \delta[\omega-\varpi(\mathbf{k})] d k_{x} d k_{y} d \omega,
$$

where $\delta[\cdot]$ denotes the Dirac's delta. Taking into account Equation (2), the band pass filter is centered on the shifted $(\mathbf{k}, \omega)$ components due to the effect of the current of encounter $\mathbf{U}$. The factor $k^{\beta}$ is the so-called modulation transfer function (MTF) $[7,16]$. The exponent $\beta$ was empirically obtained for the case of the marine radar (e.g., grazing incidence and horizontal polarization) [7], achieving a value of $\beta \approx-1.2$. This MTF was empirically obtained to make the $1 \mathrm{D}$ frequency spectrum appear more like an in-situ buoy [7]. Mention that some recent results comparing marine radar with measurements acquired by air-borne LIDAR indicate that the MTF may not be needed [22]. The results obtained in this study use the empirical MTF with the exponent $\beta$ indicated above. A detailed description of the inversion modeling technique can be consulted in $[4,7,8]$. It is known that the sea clutter values are related to the electromagnetic backscatter of the sea surface rather than the wave elevation [7]. So, the wave spectrum estimated from the analysis of marine radar image sequences, $\hat{F}(\mathbf{k}, \omega)$, is not properly scaled. It involves that Equation (4) cannot be directly applied to obtain $H_{s}$ from $\hat{F}(\mathbf{k}, \omega)[9]$. The solution used for estimating $H_{s}$ came form the existence of the BGN components in the image spectrum $I(\mathbf{k}, \omega)$ (see Figure 4$)$. This phenomenon also appears in other radar sensors used in oceanography, such as space-borne SAR systems [10]. 


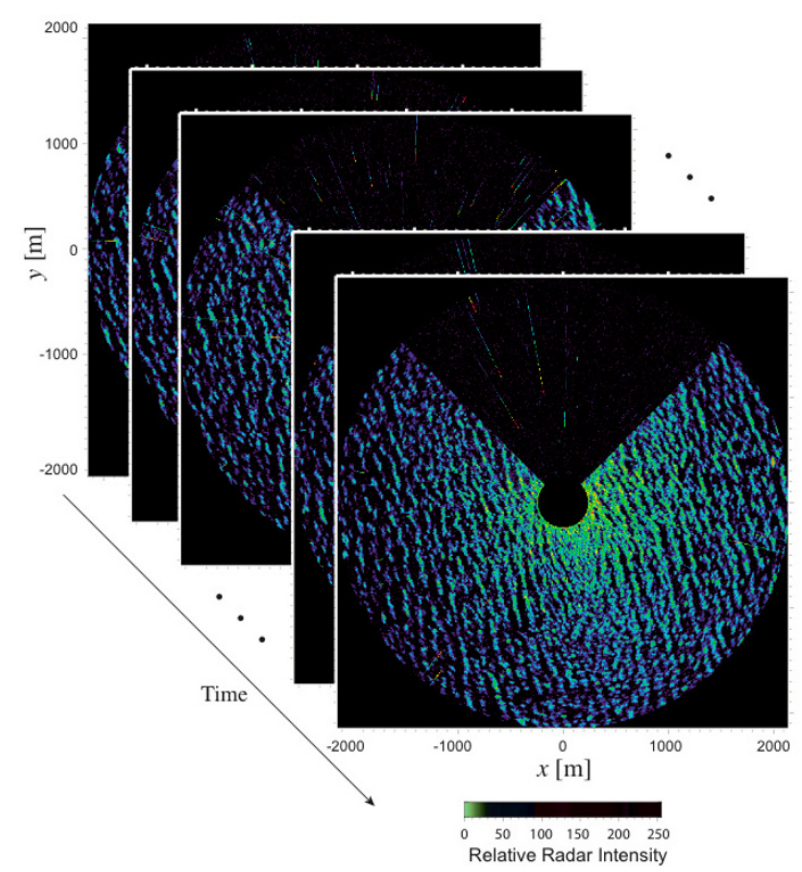

Figure 3 Sequence of marine radar images of the sea surface measured by the WaMoS II system. This sequence was acquired at the FINO 1 research platform.

As a conclusion, $H_{s}$ is estimated using a $3 \mathrm{D}$ generalization of the method proposed when processing SAR images $[9,19]$ for a given time instant, $n$, as

$$
H_{s}[n]=c_{0}+c_{1} \sqrt{\mathrm{SNR}}[n],
$$

where $c_{0}$ and $c_{1}$ are calibration constants that depend on every marine radar installation [4]. Therefore, as a part of the set up of a marine radar station to estimate $H_{s}$, it is necessary to carry out a calibration campaign using an in-situ sensor (e.g., a buoy) to determine these calibration constants. The tuning of these constants is made by least squares method. The term SNR in Equation (9) represents the ratio between the signal of the spectral energy of the unscaled wave spectrum $(\hat{F}(\mathbf{k}, \omega))$

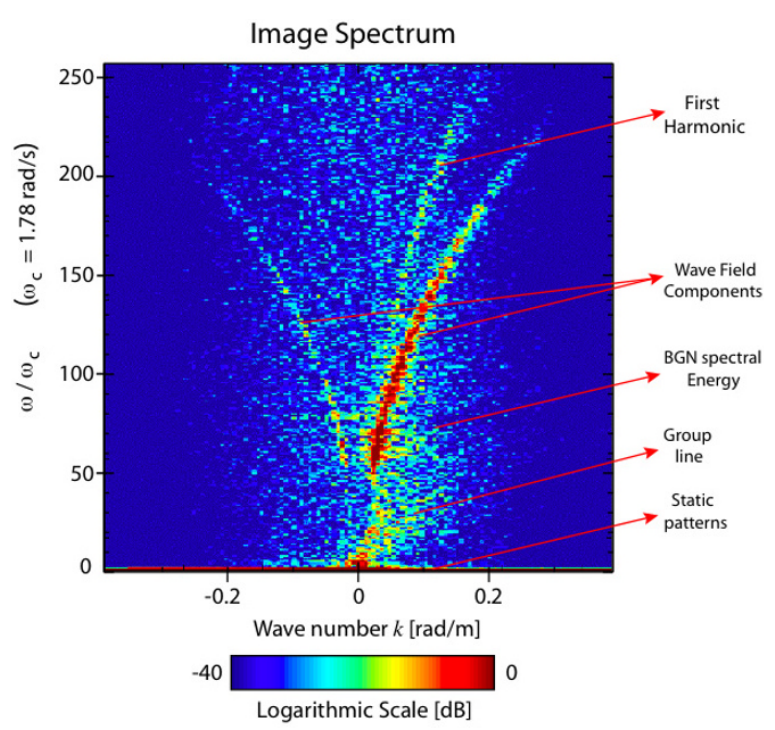

Figure 4 Example of a $2 D$ image spectrum $I(k, \omega)$, and its different spectral components $[7,9]$. A detailed description of these components can be found in $[19,21]$. 
obtained by the inversion modeling technique and the noise of the total spectral energy of the BGN components [9].

\subsection{Limitations of the standard method to estimate $\boldsymbol{H}_{\boldsymbol{s}}$} The $H_{s}$ estimation from Equation (9) provides, in general, good agreements with the results derived from in-situ sensors. This is valid while there is a minimum amount of local wind to induce enough sea surface roughness and, therefore, enough radar backscatter intensity $[4,9,23]$. However, under weak local wind conditions, the $B G N$ energy is low, giving a high value of $\sqrt{\mathrm{SNR}}$. Therefore, there is an overestimation of $H_{s}$ using Equation (9). It is known that, at grazing incidence, as marine radars work, shadowing is one of the most important modulation mechanisms of the radar imagery $[7,21]$. In addition, the wave slopes affect the backscatter mechanisms because they change the local angle of incidence of the electromagnetic field [16]. This mechanism is called tilt modulation. Both shadowing and tilt modulation depend on the wave heights and lengths [24]. Hence, additional parameters to the $\sqrt{\mathrm{SNR}}$ should be considered in the $H_{s}$ estimation. A possible solution would be including the wind speed measured by an additional sensor, like an anemometer. Nevertheless, in some radar installations, there is no wind measurements available that could give an idea about the accuracy of the $H_{s}$ estimation. However, as mentioned above, there are some sea state parameters derived from the wave spectra that depend indirectly on wind conditions. So, when the wind is low, long waves with long periods are expected (e.g., swell-dominated sea states). In these situations, nothing useful is observed. On the contrary, when the wind speed is high, short wave lengths and periods are observed (e.g., wind sea-dominated sea states). In these situations, both swell- and wind-dominated sea clutter can also be observed.

Figure 5 shows two scatter plots (one for each research platform) of the $H_{s}$ measurements using a buoy and the corresponding marine radar-based estimation using Equation (9). These measurements are given for the same time instants as in Figure 2. The dots in both plots are colored depending on the values of the peak wave length, $\lambda_{p}$, which are derived from the wave number spectrum $(\bar{F}(\mathbf{k}))$ estimated by each WamoS II sensor. $\bar{F}(\mathbf{k})$ was obtained by applying Equation (5) to the un-scaled wave spectrum $\hat{F}(\mathbf{k}, \omega)$ defined in Equation (8). For the case of Ekofisk (see Figure 5a), it can be observed that there is a structure of the scatter plot depending on $\lambda_{p}$. Therefore, although the correlation coefficient of the scatter plot is high (e.g., 0.95), there is a spread of the dots, where in most of the cases, the overestimation appears for cases where $\lambda_{p} \gtrsim 200 \mathrm{~m}$. In the case of FINO 1 (see Figure $5 \mathrm{~b}$ ), the dependence with $\lambda_{p}$ is more evident, mainly for $H_{s} \leqslant 2 \mathrm{~m}$, where the $H_{s}$ radar estimation using Equation (9) can be more than twice the value provided by the buoy, especially for long wave lengths $\left(\lambda_{p} \geq 200 \mathrm{~m}\right)$.

\section{Estimate of the significant wave height by multilayer perceptrons}

Taking into account the results presented in Section 3 (see Figure 5), the $H_{s}$ estimation derived by Equation (9)
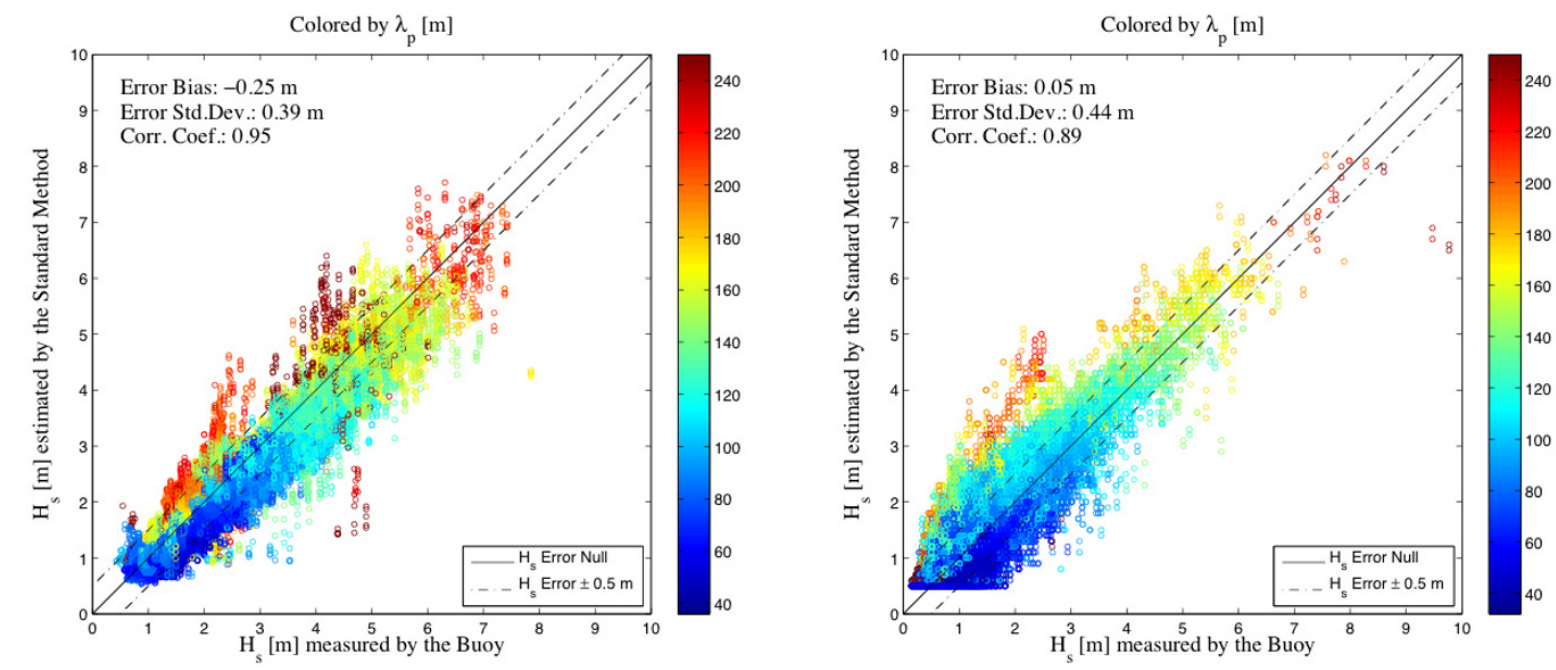

Figure 5 Scatter plots colored by $\lambda_{\mathrm{p}}$ of the $H_{s}$ measured by the buoy and the corresponding estimation provided by the WaMoS II systems mounted at Ekofisk and FINO1 platforms. The solid line represents the location where the values provided by the buoy are equal to the radar estimation. In addition, the dashed lines indicates the locations where the difference between the buoy and the radar estimation is $\pm 0.5 \mathrm{~m}$. 
should be improved to obtain a more robust estimator. This improved estimator should consider additional parameters of the wave field related to the wave length and period, which depend on the wind conditions. The solution proposed here considers not only the $\sqrt{\mathrm{SNR}}$, as done in the standard method, but also two additional parameters related to the wave length and period, such as $\lambda_{p}$ and $T_{m}$. There is no indication that $H_{s}$ presents a linear dependence on $\sqrt{\mathrm{SNR}}, \lambda_{p}$ and $T_{m}$. Therefore, and since ANNs are able to implement non-linear functions [11,25], a non-linear solution of the problem based on ANNs is investigated in this article. But, when proposing a solution using ANNs, several questions arise. In our case study, we need to know before selecting a type of ANN:

- What kind and how many sea state parameters we should consider as input.

- What kind of ANN architecture we should select, determining the type of ANN, the activation function of the ANN neurons and the ANN size.

- Once the ANN architecture is selected, which learning algorithm we should use to train it.

- Finally, to design and test the proposed ANN-based solution, how the database of $H_{s}$ measurements should be divided to correctly train the ANN and to get the best results in a testing stage.

The following sections present the answers to these questions.
4.1. MLP-based Hs estimator: architecture, data processing, and computational cost

This section presents the proposed ANN-based $H_{s}$ estimator, discussing what kind and how many sea state parameters are considered, and what ANN architecture (type, activation functions and size) is selected. The way the ANN processes the data and the computational cost of the proposed solution are presented at the end of the section.

The proposed ANN-based $H_{s}$ estimator is presented in Figure 6. For comparison purposes, the standard $H_{s}$ estimator is also presented in this figure. Each estimator takes the corresponding sea state parameters given by the WaMoS II software. This figure summarizes the architecture (type, activation functions, and size) of the ANN selected in our case of study, as well as the way the data is processed.

The kind and number of sea state parameters to be presented as input to the ANN were justified at the beginning of Section 4 . The values of these parameters at a certain instant $n$ are incorporated in the observation vector presented at the ANN input as:

$$
\mathbf{x}[n]=\left[\sqrt{\operatorname{SNR}}[n], \lambda_{p}[n], T_{m}[n]\right] .
$$

Note that $n$ represents the time scale of the sea state temporal evolution, as depicted in Figure 2, and not the time scale $(t)$ of the temporal sequence of the radar images. In other words, the estimates made by the ANN

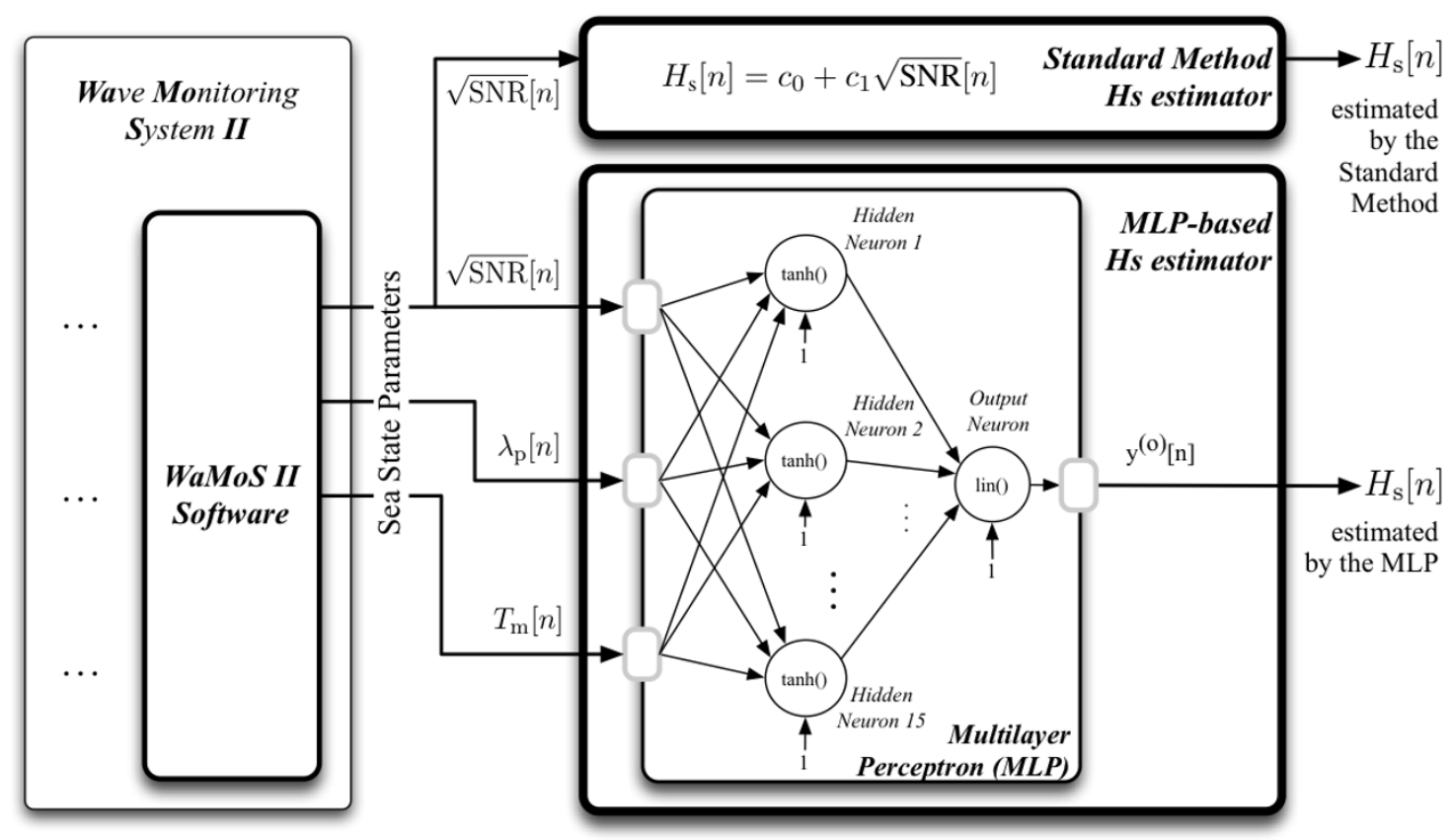

Figure 6 Scheme of the ANN-based $H_{s}$ estimator proposed in this research. 
are given at the same time instants as the measurements given by the buoy.

As indicated in Figure 6, the estimate of the $H_{s}$ is obtained directly from the output of the ANN as:

$$
H_{s}[n]=\gamma^{(o)}[n]=f_{\text {ANN }}(\mathbf{x}[n]),
$$

where $f_{\mathrm{ANN}}()$ denotes the input-output mapping function implemented by the ANN. The ANN is designed to give outputs between 0 and 10 . Note that the upper limit set for the estimated $H_{s}$ is greater than the maximum value of $H_{s}$ measured by the buoy for both platforms (see Figure 2). In this way, we try to mitigate underestimates of very high values of $H_{s}$.

Once knowing the kind and number of parameters to be used in the ANN-based $H_{s}$ estimator, the next step consists on selecting its architecture. This selection determines how the ANN processes the observation vector, and, in consequence, the way the function of Equation (11) is implemented. As noted at the beginning of the current section, a non-linear relationship between the selected sea state parameters and $H_{s}$ is investigated to make accurate $H_{s}$ estimates. ANNs can implement this non-linear function. Exactly, they can be implemented by MLPs [25], a kind of feedforward ANN. MLPs are able to learn from a pre-classified database of measurements [11]. In this way, they can implement a proper non-linear function between the input space (sea state parameters space) and the output space $\left(H_{s}\right.$ space). As an example, multilayer perceptrons (MLPs) were satisfactorily used in $[12,13]$ as sea clutter reduction systems when working with non-coherent marine radar image sequences. In this case, MLPs were used as non-linear filters to adapt the filtering to the non-linear properties of the sea clutter, i.e., they were able to implement non-linear functions. For this reason, an MLP is considered as the type of ANN used in our experiments.

Once the ANN type is selected, its size is studied. An MLP structure with two layers (input, not computed as a layer, hidden and output layers) is selected because it is demonstrated to be enough to solve a lot of kind of problems [25]. The number of MLP inputs in the input layer corresponds to the number of sea state parameters selected for this study, i.e., three inputs, being summarized in Equation (10). The number of hidden neurons in the hidden layer is selected according to the following criteria: if a few hidden neurons are selected (4, as an example), poor performance is obtained after training; but if a lot of hidden neurons are selected (50, as an example), a high risk of over-fitting the training data set exists. In this way, an intermediate number of hidden neurons should be selected. As an example, in [26], where MLPs were used to create a ship detection system, the best number of hidden neurons, considering a trade-off between performance and computational cost, was 10. Empirical studies made during our research allow us to determine that no much better performances are obtained from 15 hidden neurons for both platforms, but a computational cost increase is observed. Therefore, 15 hidden neurons are selected. Finally, one output neuron is selected because only one output is needed in the proposed system to give an estimate of the $H_{s}$. As a conclusion, the selected MLP has a structure 3/15/1.

Once set the ANN type and size, the signal processing made by the MLP for a given observation vector is presented. This signal processing is summarized in Equation (11) and is computed in two steps.

In the first step, consider $\mathbf{v}^{(\mathrm{h})}[n]$ is a vector of size $[1 \times$ 15], which elements are the inputs of each hidden neuron. Also, consider that $\mathbf{y}^{(\mathrm{h})}[n]$ is a vector of size $[1 \times 15]$ that stores the outputs of each hidden neuron. And finally, consider $\mathbf{W}^{(\mathrm{h})}$ is a matrix of size [3 $\left.\times 15\right]$, which contains the synaptic weights that connect the MLP inputs with the MLP hidden neurons, and $\mathbf{b}^{(\mathrm{h})}$ is a row vector of size [1 $\times$ 15], which contains the hidden neuron biases. According to them, Equation (12) and Equation (13) show how $\mathbf{v}^{(\mathrm{h})}$ and $\mathbf{y}^{(\mathrm{h})}$ are computed. The hyperbolic tangent activation function $(\tanh (\cdot))$ is used in this layer because it allows accelerating the learning process with respect to linear functions [11].

$$
\begin{aligned}
\mathbf{v}^{(\mathrm{h})}[n]=\mathbf{x}[n] \mathbf{W}^{(\mathrm{h})}+\mathbf{b}^{(\mathrm{h})} & \\
\mathbf{y}^{(\mathrm{h})}[n]=\tanh \left(\mathbf{v}^{(\mathrm{h})}[n]\right) & =\frac{\sinh \left(\mathbf{v}^{(\mathrm{h})}[n]\right)}{\cosh \left(\mathbf{v}^{(\mathrm{h})}[\mathrm{n}]\right)} \\
& =\frac{\mathrm{e}^{\mathbf{v}^{(\mathrm{h})}[\mathrm{n}]}-\mathrm{e}^{-\mathbf{v}^{(\mathrm{h})}[\mathrm{n}]}}{\mathrm{e}^{\mathbf{v}^{(\mathrm{h})}[\mathrm{n}]}+\mathrm{e}^{-\mathbf{v}^{(\mathrm{h})[\mathrm{n}]}}}
\end{aligned}
$$

In the second step, consider $v^{(0)}[n]$ is a variable that contains the addition of the weighted signals present at the input of the output neuron, as presented in Equation (14). Moreover, consider $\mathbf{w}^{(\mathrm{o})}$ is a column vector of size $[15 \times 1]$ that contains the synaptic weights between the hidden neuron outputs and the output neuron input, and $b^{(\mathrm{o})}$ denotes the bias of the output neuron. The output of the output neuron $\left(y^{(\mathrm{o})}[n]\right)$ is computed by Equation (15), where a linear activation function (lin $(\cdot))[11]$ is used.

$$
\begin{aligned}
& v^{(\mathrm{o})}[n]=\mathbf{y}^{(\mathrm{h})}[n] \mathbf{w}^{(\mathrm{o})}+b^{(\mathrm{o})} \\
& y^{(\mathrm{o})}[n]=\operatorname{lin}\left(v^{(\mathrm{o})}[n]\right)=v^{(\mathrm{o})}[n]
\end{aligned}
$$

Note that this layer uses a linear activation function instead of a non-linear function, as the hyperbolic function used in the hidden neurons. It is due to during the development of the research, saturation in the $H_{s}$ estimate was observed in the lower and upper limits of the 
hyperbolic function, which correspond to low and high values of $H_{s}$. This saturation is avoided using a linear activation function.

Finally, an analysis of the computational cost of the proposed solution is given. The number of operations needed to implement the MLP are given in [27] for a general MLP structure of $J / K / 1$. Therefore, for our particular case of study (structure 3/15/1), a total of 76 memory cells (accesses to memory), 60 two-element sums and 60 two-element products are needed to implement it. Unfortunately, the computational cost of the solution is greater than the one needed for implementing the solution given by the standard method (one product and one sum). Nevertheless, the computational cost of the solution is still so low that real-time is not compromised when implementing it in a standard personal computer. Execution times will be reported in Section 5 .

\subsection{MLP-based Hs estimator: learning algorithm}

Once the architecture of the ANN is set, the learning algorithm used to train the MLP must be selected. In the literature, it has been demonstrated that an MLP trained by a supervised learning algorithm to minimize the mean squared error works properly in a wide variety of problems $[11,25]$. This error is computed in the $k$ thiteration of the algorithm for a set of $\mathrm{M}_{\text {Train }} H_{s}$ measurements as:

$$
e_{\mathrm{MS}}[k]=\frac{1}{M_{\text {Train }}} \sum_{n=1}^{M_{\text {Train }}} \frac{1}{2}\left(\gamma^{\mathrm{O}}[n]-H_{\mathrm{s}}^{\text {Buoy }}[n]\right)^{2} .
$$

Since supervised learning algorithms work properly, one of them must be selected. In this case, the error back-propagation learning algorithm is selected to train the MLP [25]. But, to make the training faster, a modified version of this algorithm is used, which incorporates an adaptive learning rate and momentum [25]. This modified version of the learning algorithm was successfully used in $[12,13,26,27]$ for different purposes. This modified learning algorithm allows automatically updating the weights $\left(\mathbf{W}^{(\mathrm{h})}\right.$ and $\left.\mathbf{w}^{(\mathrm{o})}\right)$ and biases $\left(\mathrm{b}^{(\mathrm{h})}\right.$ and $\left.b^{(\mathrm{o})}\right)$ of the MLP in each algorithm iteration in a fast and stable way. An off-line actualization of the weights and biases is used for training [25]. And, in order to avoid the over-fitting of the designing data set during the MLP training, an external validation [25] of the training process is also carried out. This external validation is useful to stop the training when the mean squared error evaluated for a data set not used in the adaptation of the MLP weights and biases (validation data set) is increasing for consecutive algorithm iterations. In this way, the capability of generalization of the MLP to work with data sets never processed during the training stage is maintained.

\subsection{Division of the databases for designing and testing the MLP-based Hs estimator}

Since a supervised learning process is used to design the MLP of the proposed solution (train with external validation), we need the measurements of $H_{s}$ from a reference sensor. These measurements are taken from the buoys moored in the vicinity of the two platforms under study (Ekofisk and FINO 1). The measurements made by the buoy in these platforms were presented in Figure 2. But, according to the learning process presented in the section 4.2 , the data of each platform must be divided in three data sets. The first and second data sets are used in the designing stage of the standard method (tuning of the calibration constants of Equation (9)) and MLP-based $H_{s}$ estimators. The third data set is used in a testing stage. These data sets have different purposes, being:

- Training data set (designing stage): It is used in the training process of the, in which its synaptic weights and biases are updated to minimize the mean squared error of this data set.

- Validation data set (designing stage): It is used during the external validation process done in the MLP learning process. This data set allows estimating how the learning process is evolving and stopping the learning process in a suitable stage where the generalization capabilities of the MLP are maintained. So, we avoid the specialization of the MLP in the training data set.

- Testing data set (testing stage): This data set is used to estimate the performance of the proposed MLP-based $H_{s}$ estimator once designed and working autonomously.

Next, the division of the available databases of $H_{s}$ measurements is discussed. Three main principles drive this division:

- The first principle is based on the quantity of data that will form each data set. In this way, an equitable principle (one third) is used for the division of the database in three data sets.

- The second principle establishes that the first part of the database is dedicated to the designing stage and the last part to the testing stage. It is done in this way because we want to observe how the system will work for future estimates, i.e., once it is working autonomously.

- And the third principle is based on the information contained in each data set of the designing stage. In this way, and based on preliminary empirical studies done during our research, to obtain high performance MLPs once trained, it is needed that the data 
from the worst sea state (the highest $H_{s}$ ) are contained in the training data set.

Applying these principles to the Ekofisk and FINO 1 databases, we obtain the divisions plotted in Figure 7. Note that the estimates made by the standard method are also plotted, which will be used for comparison purposes in the results reported in Section 5. This figure plots the beginning and ending dates of each data set, as well as the number of measurements contained in them. As observed, the equitable factor of one third set by the first principle is modified by the other two principles, but maintaining a ratio data selected-total data very close to this factor. On the other hand, note that the order of the training and validation data sets for both database divisions is different. It is due to the maximum $H_{s}$ must be contained in the training data set, as set the third principle.

\section{Experimental results: comparison of the standard method and MLP-based Hs estimators}

This section presents the $H_{s}$ estimations made by the proposed MLP-based method for the two platforms (Ekofisk and FINO 1) considered in the study. They are compared with the measurements made by in-situ sensors (buoy). To validate the proposed method, these results are compared with the ones obtained by the standard method. The comparisons are made in the designing and testing stages. The aim of comparing the results obtained in both stages is to realize whether the performances obtained during the designing stage are maintained for a data set never processed before (testing data set) or not. In other words, we want to know, once the MLP-based estimator is designed, how the proposed method works from a point of view of performance and time of designing/execution.

\subsection{Comparison of the standard method and MLP-based Hs estimators for the Ekofisk platform}

The $H_{s}$ estimations made by the standard and MLP-based methods considering the data of the Ekofisk platform are presented first for the designing stage, and second for the testing stage.

\subsubsection{Designing stage of the MLP-based Hs estimator for the Ekofisk platform}

In this stage, the data of the training and validation data sets of the Ekofisk platform (see Figure 7a) are considered. Considering the data measured by the buoy for both data sets and applying the learning algorithm described in Section 4.2, a time plot of the estimates made by the proposed MLP-based method is depicted in Figure 8. As can be subjectively observed, these estimates approximate with high accuracy the measurements made by the buoy.

From Figure 7a and Figure 8, some limitations are observed in both methods. These limitations are clearer observed in the scatter plots (estimates Vs measurements) presented in Figure 9. Focusing on the results obtained by the standard method (see Figure 9a), a general $H_{s}$ underestimate (negative bias of the $H_{s}$ estimate error) is observed for the whole range of $H_{s}$. An opposite effect is particularly observed for sea states mainly conditioned by swell $\left(\lambda_{p} \geqslant\right.$ $200 \mathrm{~m}$ ). Focusing on the results obtained by the MLPbased method (see Figure 9b), not only better performance is observed in general (null bias of the $H_{s}$ estimate error), but also better performance is observed particularly for

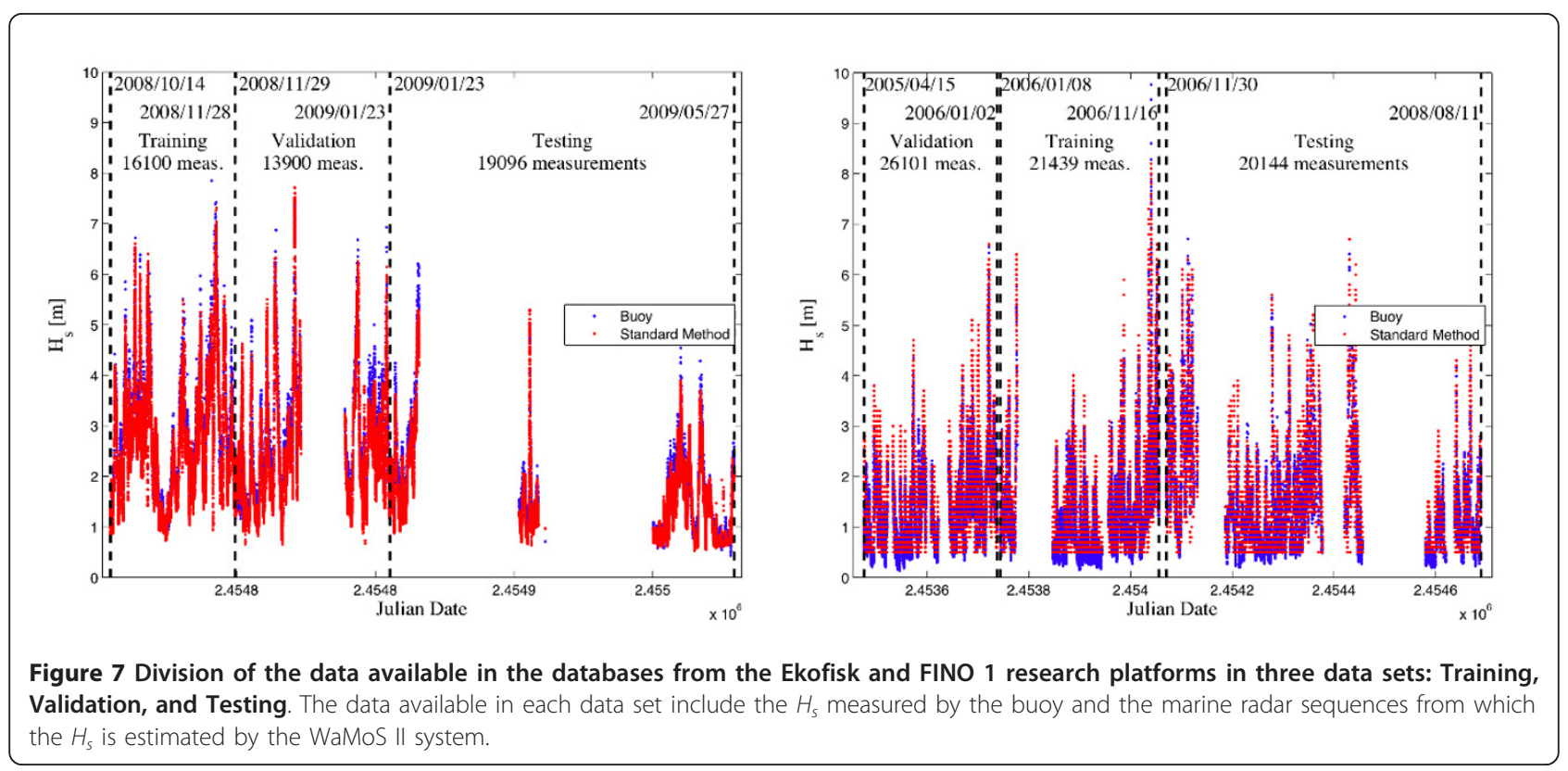




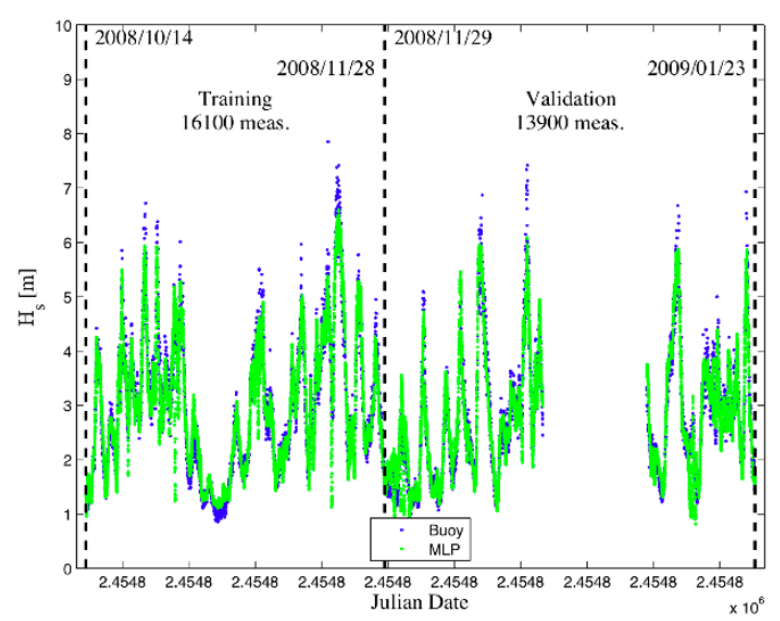

Figure 8 Temporal evolution of the $H_{s}$ estimate made by the MLP-based method for the training and validation data sets of the Ekofisk database.

sea states mainly conditioned by swell $\left(\lambda_{\mathrm{p}} \gtrsim 200 \mathrm{~m}\right)$. Apart from these general and particular improvements, its general performance is also improved because more accurate estimates are obtained, being it denoted by the reduction of standard deviation (SD) of the $H_{s}$ estimate error (from $0.44 \mathrm{~m}$ to $0.35 \mathrm{~m}$ ) and the increase of the correlation coefficient between estimates and measurements (from 0.93 to 0.96). But, there are still two limitations in the proposed approach. First, there are still some outliers in the $H_{s}$ estimates. These outliers are observed for bimodal sea states (swell and wind-generated waves are strong) with very high $\lambda_{p}$ values, being observed for $H_{s} \in[4.5,5.0] \mathrm{m}$. These outliers concern only to a few data of the designing data sets, being it the reason why the MLP is not properly learning from the environmental conditions of these data.
In other words, the MLP is learning from the environmental conditions of the majority of the data. And second, there are still some $H_{s}$ underestimations, but they are lower in number than for the standard method and not predominant because the mean error of the $H_{s}$ estimate is close to $0 \mathrm{~m}$.

\subsubsection{Testing stage of the MLP-based Hs estimator for the Ekofisk platform}

Once the standard method and MLP-based $H_{s}$ estimators are designed, and they are autonomously working, we analyze whether the performances and limitations discussed above continue being present or not using a new data set, the testing data set. A time plot of the $H_{s}$ measured by the buoy and estimated by the standard method for the testing data set was presented in Figure $7 \mathrm{a}$,
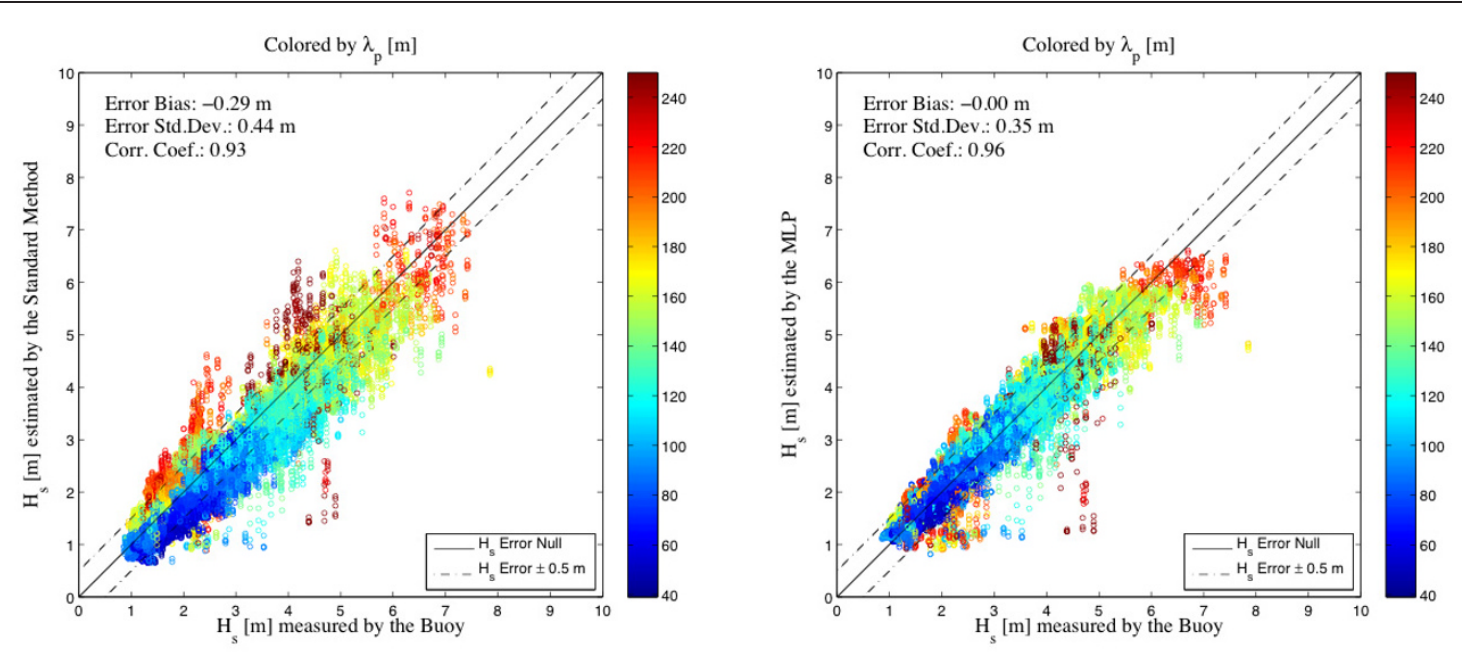

Figure 9 Scatter plots of the $H_{s}$ measured by the buoy and estimated by the standard method or proposed MLP-based estimators for the training and validation data sets of the Ekofisk database. 
whereas for the $H_{s}$ estimated by the MLP-based approach is depicted in Figure 10.

As occurred in the designing stage, some limitations are observed from Figure $7 \mathrm{a}$ and Figure 10, which can be better observed in the scatter plots of Figure 11. Focusing on the estimates obtained by the standard method (see Figure 11a), the limitations of this method previously observed for the training and validation data sets are endorsed. In this way, an $H_{s}$ overestimate is observed for swell-dominated sea states $\left(\lambda_{\mathrm{p}} \geq 200 \mathrm{~m}\right)$ and an $H_{s}$ underestimate is observed in general (negative bias of the $H_{s}$ estimate error). Focusing on the estimates obtained by the MLP-based method (see Figure 11b), most of the conclusions obtained in the designing stage are endorsed in this stage, but with some differences. In this case, a low overestimate of $H_{s}(+0.14 \mathrm{~m})$ is obtained in general, what did not happen in the designing stage. But, the problem of overestimating $H_{s}$ for swell-dominated sea states $\left(\lambda_{\mathrm{p}} \gtrsim 200 \mathrm{~m}\right)$ continues being solved, being clearly observed in the region of $H_{s} \in[0.5,1.0] \mathrm{m}$. The second difference concerns to the high reduction rate of outliers. The third difference concerns to the high concentration of $H_{s}$ estimates close to the line of null error and between the lines denoting an $H_{s}$ error of $\pm 0.5 \mathrm{~m}$. This high concentration of estimates denotes high accuracy in the estimates, improving the results obtained by the standard method. This improvement can be observed by the decrease of the SD of the $H_{s}$ estimate error (from $0.27 \mathrm{~m}$ for the standard method to $0.22 \mathrm{~m}$ for the MLP-based method) and the increase of the correlation coefficient (from 0.95 for the standard method to 0.97 for the MLP-based method). The last difference concerns to the presence of $H_{s}$ underestimations, which presence is practically negligible in the test case.

\subsection{Comparison of the standard method and MLP-based Hs estimators for the FINO 1 platform}

As done for the case of study of the Ekofisk platform, a study of the performances of the $H_{s}$ estimators based on the standard method and MLPs is made in the designing and testing stages.

\subsubsection{Designing stage of the MLP-based Hs estimator for the FINO 1 platform}

In this stage, the data of the training and validation data sets of the FINO 1 platform are considered (see Figure $7 \mathrm{~b}$ ). The $H_{s}$ measured by the buoy for these data sets is used in the MLP learning algorithm described in Section 4.2. A time plot of the estimates made by the proposed MLPbased method for both data sets is plotted in Figure 12. Comparing both figures, we observe that the estimates made by the proposed method approximate the measurements made by the buoy with an accuracy higher than the one obtained by the standard method.

The accuracy mentioned above can be clearer observed in the scatter plots of Figure 13. Focusing on the estimates obtained by the standard method, we observe that, even when the bias of the $H_{s}$ estimate error is close to null, there are still some over and underestimates present. So, comparing these results with the results obtained for the Ekofisk platform (see Figure 9a and Figure 13a), it is observed that poorer estimations are made. It can be objectively observed by the decrease of the correlation coefficient of the temporal series $(0.93$ for Ekofisk and 0.89 for FINO 1), while the SD of the $H_{s}$ estimate error is maintained ( $0.44 \mathrm{~m}$ for both platforms). It is important to note that, as occurred for the Ekofisk platform, an overestimation of $H_{s}$ is still made for swelldominated sea states $\left(\lambda_{\mathrm{p}} \gtrsim 200 \mathrm{~m}\right)$, as observed in Figure 13a. On the other hand, and focusing on the results obtained by the MLP-based method (see Figure 13b), a

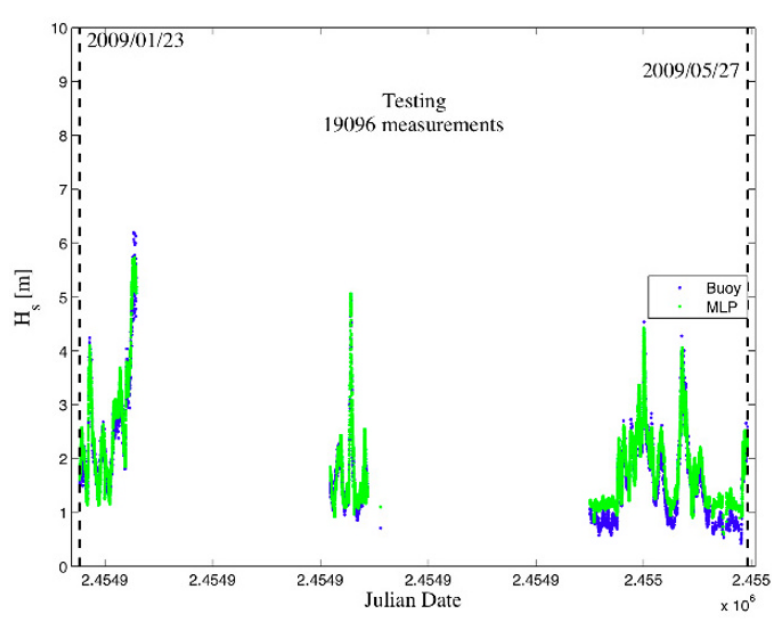

Figure 10 Temporal evolution of the $H_{s}$ estimate made by the MLP-based method for the testing data set of the Ekofisk database. 

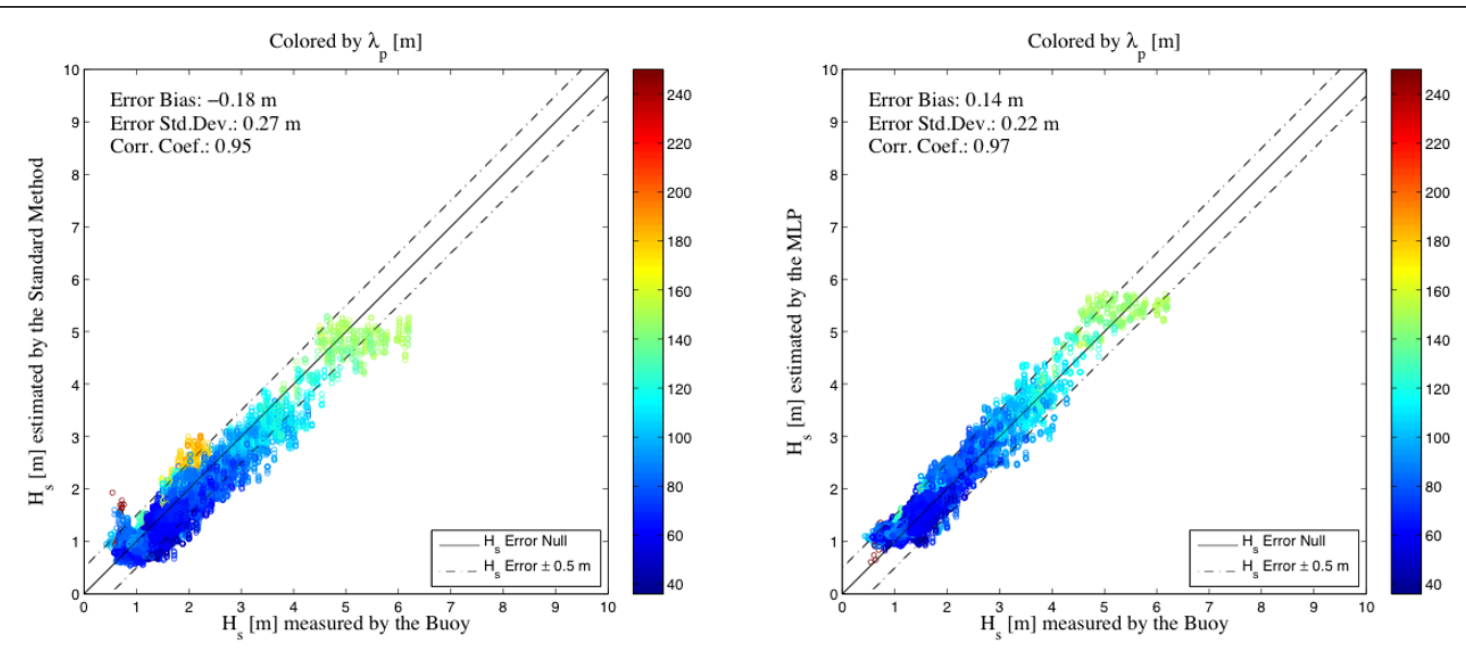

Figure 11 Scatter plots of the $H_{s}$ measured by the buoy and estimated by the standard method or proposed MLP-based estimators for the testing data set of the Ekofisk database.

performance improvement, with respect to the results achieved by the standard method, is observed in general, with a negligible bias in the $H_{s}$ error estimate. This performance improvement is subjectively observed in Figure $13 \mathrm{~b}$ because the estimates are more concentrated between the curves of $H_{s}$ error $\pm 0.5 \mathrm{~m}$. This performance improvement can be objectively observed by the reduction of the SD of the $H_{s}$ estimate error (from $0.44 \mathrm{~m}$ for the standard method to $0.24 \mathrm{~m}$ for the MLP-based method) and the increase of the correlation coefficient (from 0.89 for the standard method to 0.97 for the MLPbased method). Finally, it is also important to note that the particular problem of overestimating $H_{s}$ for swelldominated sea states is solved by the proposed method. As an example, see how the overestimates made by the standard method in the range $H_{s} \in[1.0,2.5] \mathrm{m}$ are corrected by the MLP-based method. But, there are still some $H_{s}$ underestimates present.

\subsubsection{Testing stage of the MLP-based Hs estimator for the FINO 1 platform}

This section shows the results obtained when processing a new data set of the FINO 1 platform, the testing data set. A time plot of the $H_{s}$ estimates and measurements made by the standard method and the buoy for this data set, respectively, are plotted in Figure 7b, whereas Figure 14 presents the estimates made by the MLP-based method. As can be subjectively observed from these figures, better performance is obtained by the proposed method, achieving more accurate estimates of $H_{s}$.

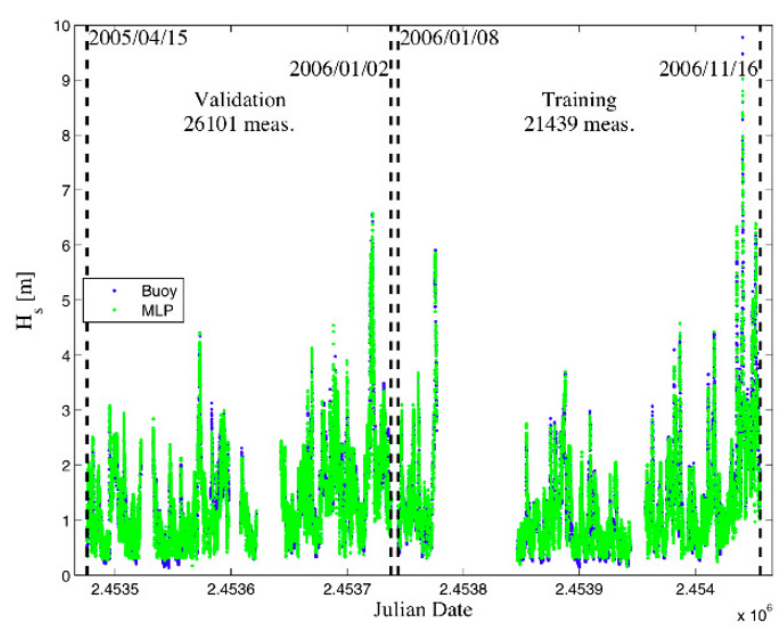

Figure 12 Temporal evolution of the $H_{s}$ estimate made by the MLP-based method for the training and validation data sets of the FINO 1 database. 

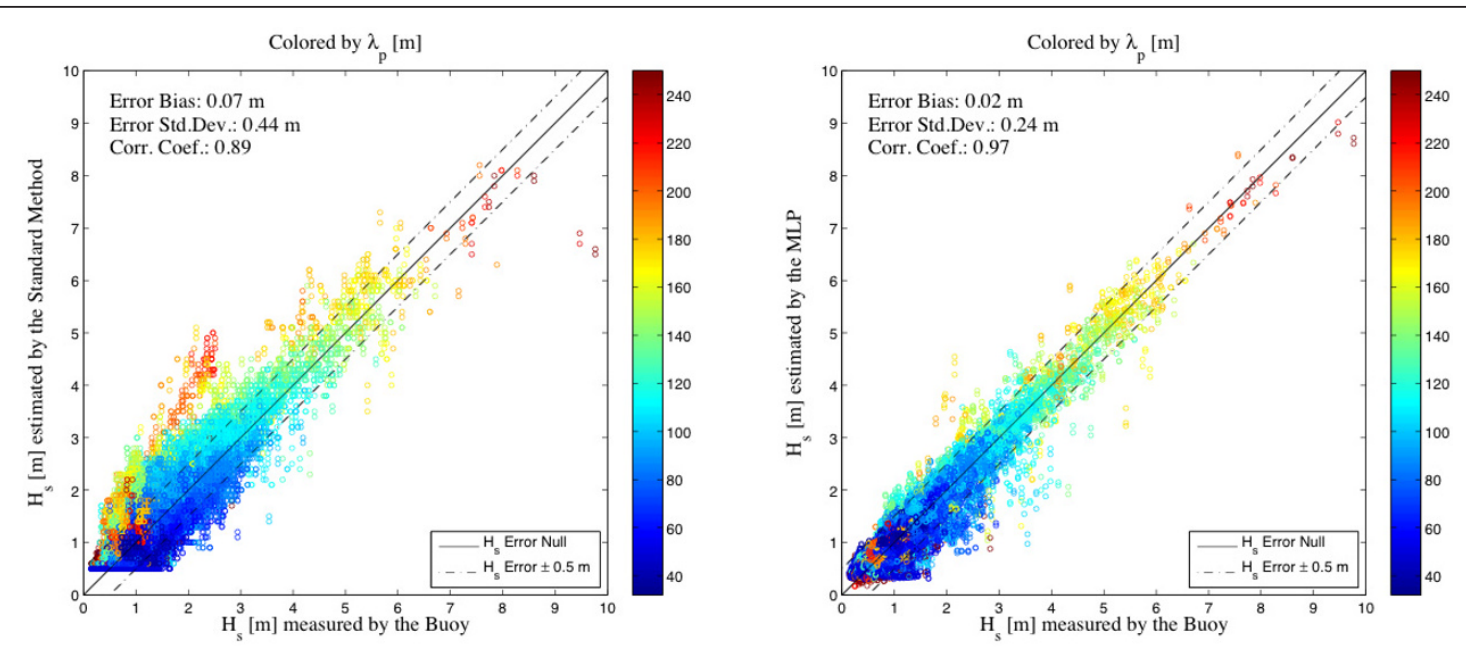

Figure 13 Scatter plots of the $H_{s}$ measured by the buoy and estimated by the standard method or proposed MLP-based estimators for the training and validation data sets of the FINO 1 database.

The above mentioned accuracy can be better observed by the scatter plots presented in Figure 15. Making an analysis as the one presented for the designing stage, similar conclusions can be obtained in the testing stage for both methods. So, considering the estimates achieved by the standard method, the statistical results given in Figure 15a for the testing data set are maintained with respect to the ones given in Figure 13a for the designing data sets. Moreover, the problem of overestimating $H_{s}$ for swell-dominated sea states is still present in the standard method. On the other hand, and considering the results achieved for the MLP-based method, a small decrease of the performance obtained in the testing stage (see Figure 15b) with respect to the one obtained in the designing stage (see Figure 13b) is observed. Moreover, no problem is observed when $H_{s}$ is estimated for swell-dominated sea states $\left(\lambda_{p} \geq 200 \mathrm{~m}\right)$. But, the problem of having a few underestimates is still present in the proposed method. As a conclusion, the advantages and limitations of both methods are endorsed when processing a new data set in the testing stage.

\subsection{Comparison of the standard and MLP-based Hs estimators for both platforms}

This section presents a comparison of the performances achieved by the standard method and MLP-based $H_{s}$ estimators when working with data from the Ekofisk or FINO 1 platforms. Since the most important aspect of the methods is to observe how they work once designed, i.e., when they are autonomously working, this comparison is made using the performances obtained for the

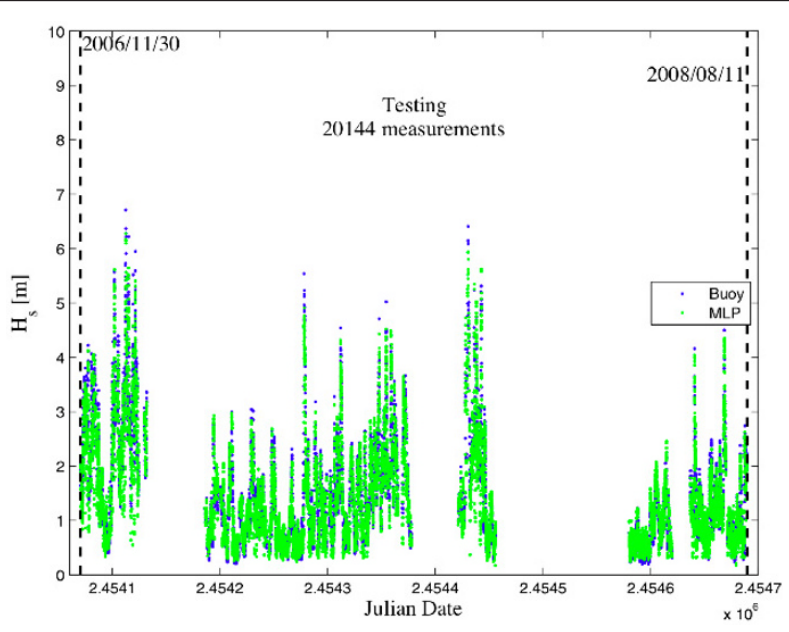

Figure 14 Temporal evolution of the $H_{s}$ estimate made by the MLP-based method for the testing data set of the FINO 1 database. 

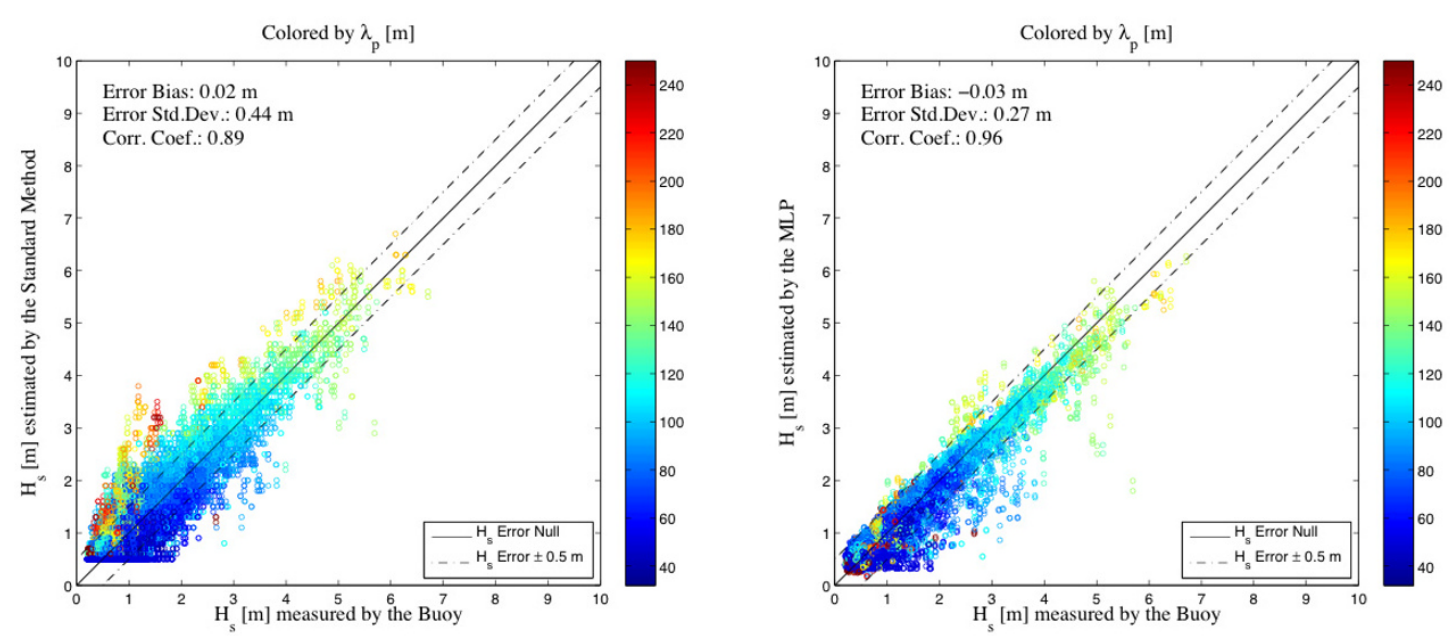

Figure 15 Scatter plots of the $H_{s}$ measured by the buoy and estimated by the standard method or proposed MLP-based estimators for the testing data set of the FINO 1 database.

testing data set. In this way, Table 2 summarizes the statistical results of the $H_{s}$ estimate error and the correlation coefficients obtained by both methods in the testing stage and for both platforms. Moreover, the performance improvements achieved by the proposed method with respect to the standard one are also given for comparison purposes.

As can be observed in Table 2, the proposed method always outperforms the standard one, regardless of the platform. Moreover, it is observed that the achieved improvement is even higher for the FINO 1 platform. But, why does it happen? As described in Section 2, this platform is located in an area of the North Sea where swell-dominated sea states are commonly present. In this way, it is observed that the proposed method works better than the standard one in this kind of sea states. Finally, it is important to note that, comparing the results obtained by the proposed MLP-based method for both platforms, the performances are similar. It denotes that the proposed method presents a great robustness against sea state changes and maintains its performance regardless of the sea state conditions where the marine radar images are obtained. It is important to note that since each non-coherent $\mathrm{X}$-band marine radar is calibrated in each site, obtaining different calibration parameters in each one during their calibration campaigns, different estimates of sea state parameters are made, such as the SNR parameter. So, the MLP-based estimator must be designed (tuned) for each radar site, as done for tuning the constants $c_{0}$ and $c_{1}$ of Equation (9) in the standard method.

Finally, the time needed for designing (training with external validation) and testing an MLP is reported for both platforms. The time values presented below are obtained implementing the designing and testing stages of the MLP-based approach in Matlab 2007a and using a standard personal computer with a $2.4 \mathrm{GHz}$ Intel Core2 Duo CPU, 4 GB of DDR2 PC2-5300 RAM and running Linux. The measured average time values are:

- Designing time of an MLP for the Ekofisk platform using the training and validation data sets of Figure $7 \mathrm{a}: \approx 30 \mathrm{~s}$ in average, considering a total of $\approx 30000$ measurements.

- Designing time of an MLP for the FINO 1 platform using the training and validation data sets of Figure 7b: $\approx 55 \mathrm{~s}$ in average, considering a total of $\approx$ 47500 measuremensurements.

- Time for processing a given measurement (vector composed of: $\sqrt{\mathrm{SNR}}, \lambda_{p}$ and $T_{m}$ ) once the MLP is

Table 2 Comparative of the statistics of the $\boldsymbol{H}_{s}$ estimates made by the standard method and proposed MLP-based estimators once designed, i.e., when processing the testing data set

\begin{tabular}{lcccccc}
\hline & \multicolumn{3}{c}{ Ekofisk } & & \multicolumn{2}{c}{ FINO 1} \\
\cline { 2 - 7 } & Error bias & Error SD & Corr. Coef. & Error bias & Error SD & Corr. Coef. \\
\hline Standard method & $-0.18 \mathrm{~m}$ & $0.27 \mathrm{~m}$ & 0.95 & $+0.02 \mathrm{~m}$ & $0.44 \mathrm{~m}$ & 0.89 \\
MLP-based method & $+0.14 \mathrm{~m}$ & $0.22 \mathrm{~m}$ & 0.97 & $-0.03 \mathrm{~m}$ & $0.27 \mathrm{~m}$ & 0.96 \\
Improvement & - & $18.5 \%$ & $2.1 \%$ & - & $38.6 \%$ & $7.8 \%$ \\
\hline
\end{tabular}


designed: $\approx 100 \mu s$ in average, regardless of the platform.

From an operational point of view, the design (train) of the MLP is proposed to be performed during the calibration campaign of the radar, when the data from the buoy are available.

\subsection{Influence of the dimensioning and composition of the designing data sets}

In the previous sections, we observed how the proposed method based on MLPs outperform the standard method when estimating $H_{s}$. For doing so, we considered large data sets for designing the MLP and high values of $H_{s}$ in them. But, what does it happen when neither the designing data sets are so large nor it incorporates high values of $H_{s}$ ? For finding an answer to this question, we reduce the number of measurements (dimensioning) considered in the designing data sets of the experiments made for each platform, and vary their composition by selecting the time instants for which the measurements do not present high values of $H_{s}$.

Starting with the measurements of the Ekofisk platform, we divide the database as presented in Figure 16. Comparing this database division with the one used originally (see Figure 7a), the following differences are found:

- Reduction of the number of measurements used in the designing data sets in approximately 70\%: from $\approx 40000$ to $\approx 12600$.

- Reduction of the maximum $H_{s}$ considered in the designing data sets in approximately $17 \%$ : from $\approx 7.8$ $\mathrm{m}$ to $\approx 6.5 \mathrm{~m}$.
Considering this new division of the Ekofisk database, we design the MLP (tuning of its parameters), as done for the original case of study, and we test it. The estimates obtained by the standard and proposed methods when processing the new testing data set are depicted in the scatter plots of Figure 17. Comparing these results with the ones obtained in the original case (see Figure 11), we observe several important aspects. First, the bias of the $H_{s}$ estimate error is very similar each other, being still very low. Second, the SD of this error is increased with respect to the original ones in both methods. Third, the correlation coefficient is maintained in both cases and methods, being very high again. Fourth, the problem of overestimating the $H_{s}$ for high values of $\lambda_{p}$ is solved again by the proposed method. Fifth, there are still some underestimations of $H_{s}$ in the proposed method, but its number is much lower than the one obtained in the standard method. Sixth, the dispersion of the measurements is greater for high values than for low values of $H_{s}$ in the MLP-based method. It happens because there were not data available of these heights in the designing data sets, but the $H_{s}$ estimation still maintains high accuracy. And seventh, since the number of measurements used now for designing MLPs is lower than in the original case, the time needed for training an MLP is reduced in $\approx 60 \%$ : from $\approx 30 \mathrm{~s}$ in the original case to $\approx 12 \mathrm{~s}$ with this new data set dimensioning. Since the size of the MLP does not vary in the experiments, the time needed for obtaining an estimate of the $H_{s}$ is the same as in the original case of study, i.e., $\approx 100 \mu s$.

Finally, we perform a similar experiment (design and test) as made above for the Ekofisk platform data, but with the data of the FINO 1 platform. In this way, we divide the database as presented in Figure 18, where we

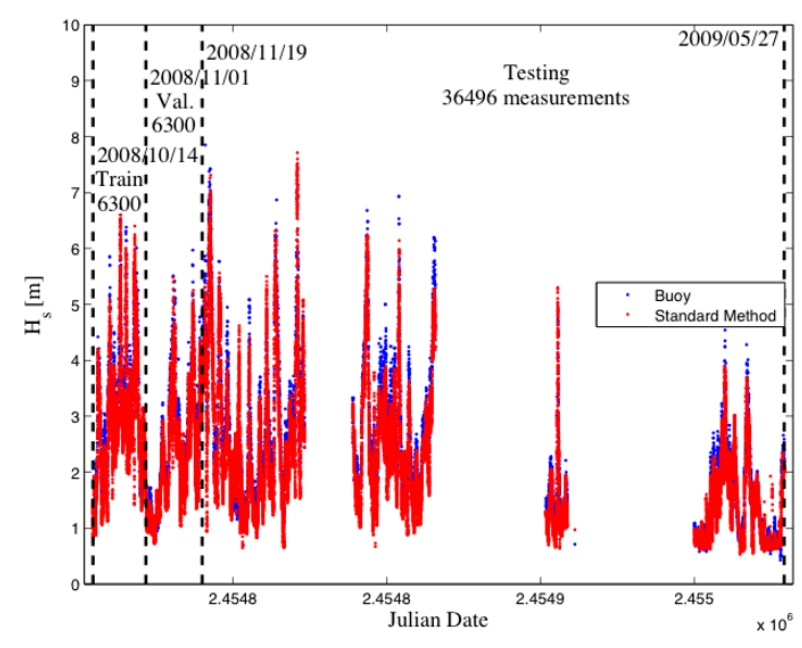

Figure 16 New dimensioning and composition of the data sets of the Ekofisk database. 

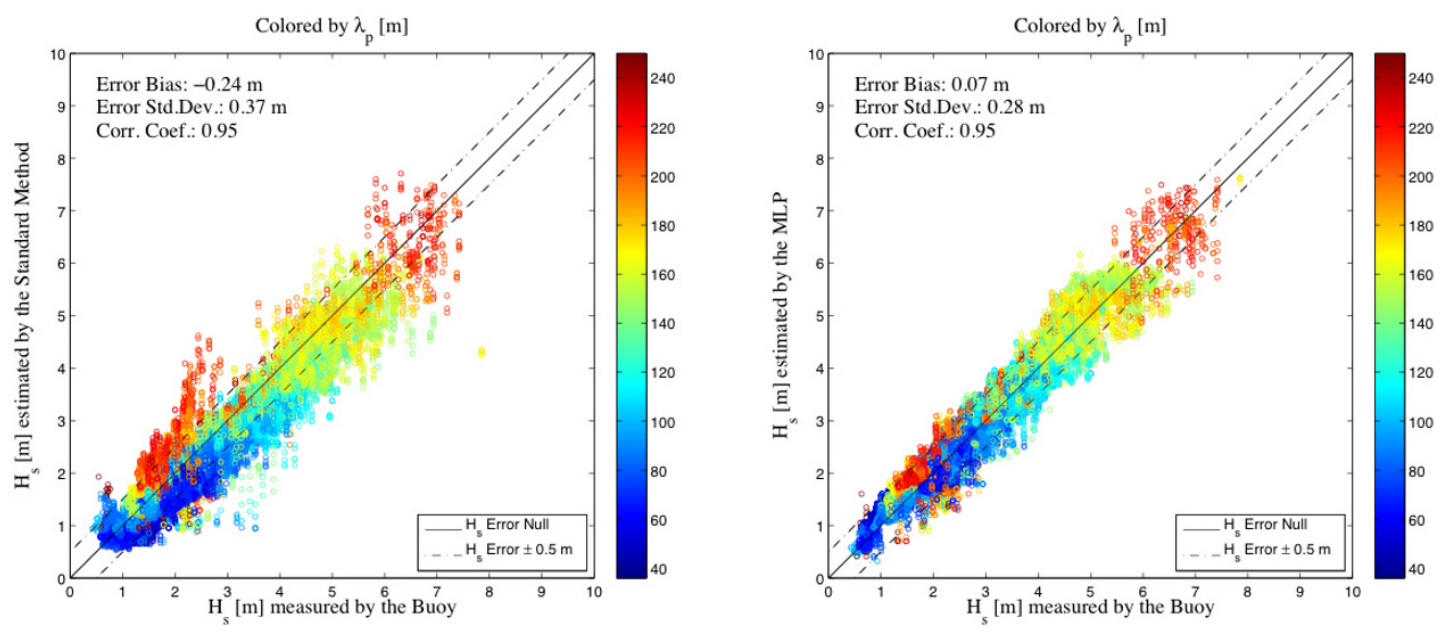

Figure 17 Scatter plots of the $H_{s}$ measured by the buoy and estimated by the standard method or proposed MLP-based estimators for the testing data set of the Ekofisk database using the data set distribution of Figure 16.

apply deeper modifications in the composition of the designing data sets with respect to the original case (see Figure $7 \mathrm{~b})$. These modifications are:

- Reduction of the number of measurements used in the design data sets in approximately $50 \%$ : from $\approx$ 47500 to $\approx 23000$.

- Reduction of the maximum $H_{s}$ considered in the designing data sets in approximately $50 \%$ : from $\approx$ $10.0 \mathrm{~m}$ to $\approx 5.0 \mathrm{~m}$.

The $H_{s}$ estimates obtained by the standard and proposed methods when processing the new testing data set of the FINO 1 platform data are depicted in the scatter plots of Figure 19. Comparing these results with the ones obtained in the original case (see Figure 15), we observe similarities and differences with respect to the aspects observed in the previous analysis performed for the Ekofisk platform. Focusing on the differences, we observe four main aspects. First, the SD of the $H_{s}$ error estimate is reduced in this case. Second, there are some underestimations for the whole range of $H_{s}$ (see Figure 19b), but they are not so strong as in the original case (see Figure $15 \mathrm{~b})$. Even when they exist, they are less in number and lower in error than the ones obtained by the standard method (compare Figure 19a and 19b). Third, the levels of dispersion and underestimation are higher in the highest range of $H_{s}$ values $\left(H_{s}>7 \mathrm{~m}\right)$. It happens because there were not available data of this kind when designing (training) the MLP. But even with that, these levels are

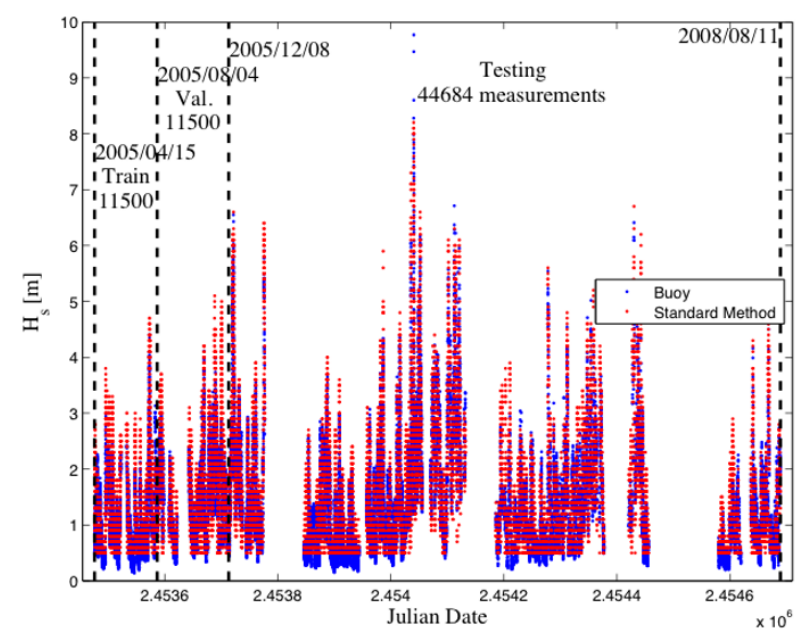

Figure $18 \mathrm{New}$ dimensioning and composition of the data sets of the FINO1 database. 

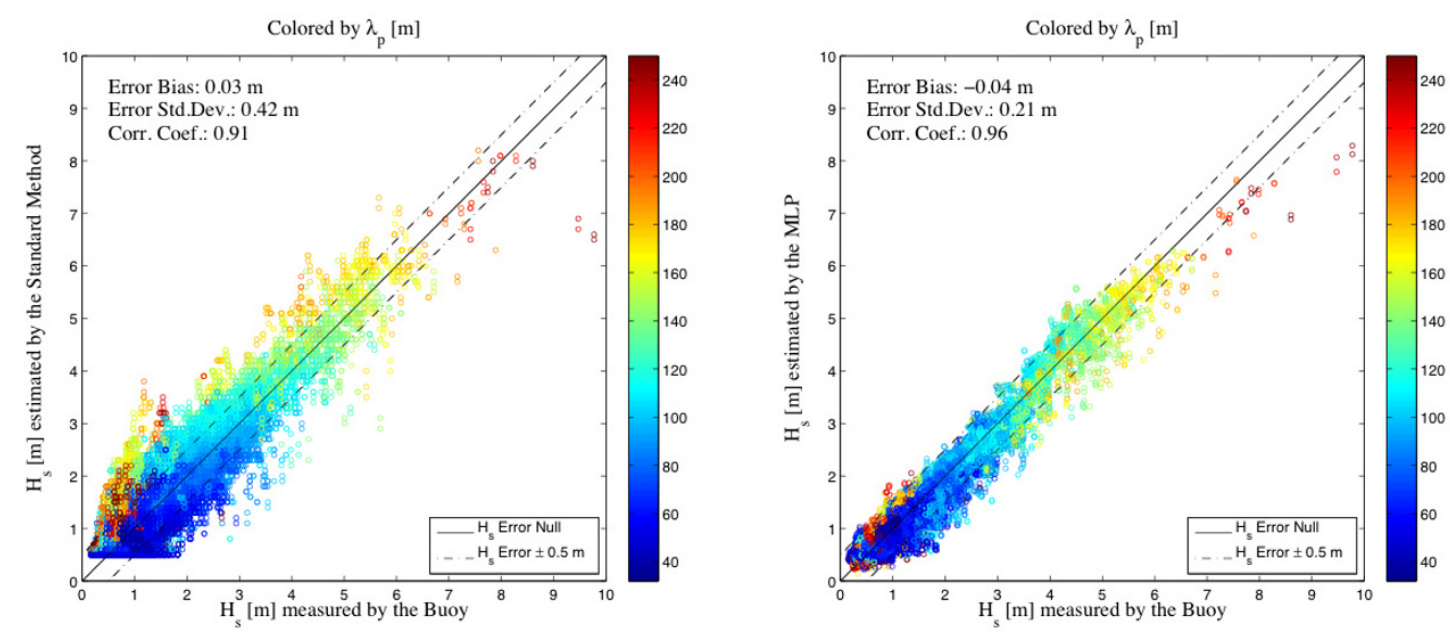

Figure 19 Scatter plots of significant wave heights measured by the buoy and estimated by the standard method or proposed MLPbased estimators for the testing data set of the FINO 1 database using the data set distribution of Figure 18.

not very high and the proposed approach is still working properly. And fourth, the time needed for training an MLP is reduced in $\approx 45 \%:$ from $\approx 55 \mathrm{~s}$ in the original case to $\approx 30 \mathrm{~s}$ with this new data set dimensioning. The time for obtaining an estimate of the $H_{s}$ is the same as in the original case and as for the other platform, i.e., $\approx 100 \mu s$.

\section{Conclusions}

A novel method for improving the $H_{s}$ estimate has been presented in this article. This method is based on the use of MLPs for implementing a non-linear function that relates the selected input parameters with $H_{s}$. The parameters selected in our case study are: the square root of the signal-to-noise ratio $(\sqrt{\mathrm{SNR}})$, the peak wave length $\left(\lambda_{p}\right)$, and the mean wave period $\left(T_{m}\right)$. The WaMoS II software used in the experiments extracts the values of these parameters from temporal sequences of radar images in real-time.

After analyzing the results achieved by the MLP-based method proposed for estimating $H_{s}$ and comparing them with the ones achieved by the standard method, four main conclusions are drawn. The first conclusion is focused on the performance improvement achieved by the proposed method. This method is able to outperform the standard method by reducing the SD of the $H_{s}$ estimate error and increasing the correlation coefficient of the $H_{s}$ time series, while maintaining a negligible estimate error bias. The second conclusion concerns to the mitigation of the problem of overestimating $H_{s}$ for swell-dominated sea states observed in the standard method. This mitigation is achieved regardless of the platform under study. The third one concerns to the robustness of the solution against sea state changes and platform. In this way, similar performances are achieved for different places of the North Sea (different platforms), where different sea states are commonly observed. This indicates that the performances presented here can be maintained for new data sets processed in the future for the same platforms. The last conclusion is focused on the low computational cost of the proposed method. Thus, once the MLP is trained, the time needed to make an $H_{s}$ estimate $(\approx 100 \mu \mathrm{s}$ in average) is much lower than the time between two consecutive $H_{s}$ estimates (minimum of $\approx 180 \mathrm{~s}$ ). In consequence, the proposed MLP-based $H_{s}$ estimator is able to process data in real-time.

\section{Acknowledgements}

This research study had been supported by Ministerio de Ciencia $e$ Innovación, MICINN, under project number TEC2009-14217. The WaMoS ॥ and buoy data obtained at the FINO 1 Platform was kindly provided by the Federal Maritime and Hydrographic Agency of Germany (Bundesamt für Seeschiffahrt und Hydrographie, BSH) and OceanWaves GmbH, Germany. In addition, OceanWaves $\mathrm{GmbH}$ kindly provided the data acquired at the ConocoPhillips Ekofisk oil field.

\section{Competing interests}

The authors declare that they have no competing interests.

Received: 11 July 2011 Accepted: 17 April 2012 Published: 17 April 2012

\section{References}

1. O Nwogu, D Lyzenga, Surface-wavefield estimation from coherent marine radars. IEEE Geosci Remote Sens Lett. 7, 631-635 (2010)

2. W Plant, W Keller, K Hayes, C Chatham, Measuring and modeling the nrcs of the sea for backscatter, in IEEE Int Geoscience and Remote Sensing Symp 2008. IGARSS 2008, Boston, 4, 65-68 (Ju 2008)

3. D Trizna, Comparisons of a fully coherent and coherent-on-receive marine radar for measurements of wave spectra and surface currents, in IEEE Oceans, Sydney, 1, 1-4 (Sep 2010)

4. K Reichert, K Hessner, J Dannenberg, I Tränkmann, B Lund, X-band radar as a tool to determine spectral and single wave properties, in Proc 5th Int Symp on Ocean Wave Measurement and Analysis, WAVES 2005, Madrid, 1 1-9 (Jul 2005) 
5. K Hessner, K Reichert, J Dittmer, JN Borge, H Güther, Evaluation of wamos ii wave data, in Proc of the Waves 2001 Conference, San Francisco, 1, 221-230 (2001)

6. P Izquierdo, J Nieto-Borge, C Guedes-Soares, R Sanz-González, R Rodríguez, Comparison of wave spectra from nautical radar images and scalar buoy data. J Waterway Port Coastal \& Ocean Eng. 131, 123-131 (2005). doi:10.1061/(ASCE)0733-950X(2005)131:3(123)

7. J Nieto-Borge, G Rodríguez, K Hessner, P Izqiuerdo, Inversion of marine radar images for surface wave analysis. J Atmos Ocean Technol. 21, 1291-1300 (2004). doi:10.1175/1520-0426(2004)0212.0.CO;2

8. F Ziemer, C Brockmann, R Vaughan, J Seemann, C Senet, Radar survey of near shore bathymetry within the oroma project. EARSeL eProc. 3, 282-288 (2004)

9. J Nieto-Borge, K Hessner, P Jarabo-Amores, D Mata-Moya, Signal-to-noise ratio analysis to estimate ocean wave heights from $x$-band marine radar image time series. IET Radar Son Nav. 2, 35-41 (2008). doi:10.1049/ietrsn:20070027

10. W Alpers, K Hasselmann, Spectral signal to clutter and thermal noise properties of ocean wave imaging synthetic aperture radars. Int J Rem Sens. 3, 423-446 (1982). doi:10.1080/01431168208948413

11. C Bishop, Neural Networks for Pattern Recognition, (Oxford University Press Inc., New York, 1995)

12. R Vicen-Bueno, R Carrasco-Álvarez, M Rosa-Zurera, JC Nieto-Borge, Sea clutter reduction and target enhancement by neural networks in a marine radar system. Sensors. 9(3), 1913-1936 (2009). doi:10.3390/s90301913

13. R Vicen-Bueno, R Carrasco-Álvarez, M Rosa-Zurera, JC Nieto-Borge, MP Jarabo-Amores, Artificial neural network-based clutter reduction systems for ship size estimation in maritime radars. EURASIP J Adv Signal Proc. 2010, $1-15(2010)$

14. I Young, W Rosenthal, F Ziemer, Radar survey of near shore bathymetry within the oroma project. J Geophys Res. 90, 1049-1059 (1985). doi:10.1029/JC090iC01 p01049

15. J Nieto-Borge, A Baquero-Martinez, D de la Mata-Moya, J Alvarez-Perez, Analysis of the sea clutter structure using temporal sequences of $x$-band marine radar images. in Int Conf on Radar, Rome, 1, 563-568 (May 2008)

16. W Plant, W Keller, K Hayes, G Chatham, Normalized radar cross section of the sea for backscatter: 1. Mean levels. J Geophys Res. 115, 11-21 (2010)

17. H Krogstad, K Trulsen, Interpretations and observations of ocean wave spectra. Ocean Dyn. 60, 973-991 (2010). doi:10.1007/s10236-010-0293-3

18. A Babanin, Breaking and Dissipation of Ocean Surface Waves (Cambridge University Press, Cambridge, 2011)

19. J Nieto-Borge, Significant wave height estimation from nautical radar data sets, (Post-doc research, GKSS Research Centre, Geesthacht, 1998)

20. J Nieto-Borge, K Hessner, K Reichert, Estimation of the significant wave height with $\mathrm{x}$-band nautical radars, in Ocean Mechanic and Arctic Engineering, OMAE-99 Saint Johns, 1, 1-8 (Jul 1999)

21. J Seemann, F Ziemer, C Senet, A method for computing calibrated ocean wave spectra from measurements with a nautical $x$-band radar, in IEEE Conf Proc Oceans 97, Halifax, 2, 1148-1154 (Oct. 1997)

22. E Terrill, A de Paolo, Phase resolved measurements of ocean waves using X-band radar, in ONR Workshop on Deterministic Measurement and Simulation of Ocean Waves, La Jolla, 1, 1-10 (Oct. 2009)

23. K Reichert, B Lund, Ground based remote sensing as a tool to measure spatial wave field variations in coastal approaches. Journal of Coastal Research. 50, 427-431 (2007)

24. C Stevens, E Poulter, M Smith, J McGregor, Nonlinear features in waveresolving microwave radar observations of ocean waves. IEEE J Ocean Eng 24(2), 470-480 (2002)

25. S Haykin, Neural Networks A Comprehensive Foundation, 2nd edn. (PrenticeHall, London, 1999)

26. R Vicen-Bueno, R Carrasco-Álvarez, MP Jarabo-Amores, JC Nieto-Borge, E Alexandre-Cortizo, Detection of ships in marine environments by square integration mode and multilayer perceptrons. IEEE Trans Instrum Meas. 60(3), 712-724 (2011)

27. R Vicen-Bueno, R Carrasco-Ávarez, MP Jarabo-Amores, JC Nieto-Borge, M Rosa-Zurera, Ship detection by different data selection templates and multilayer perceptrons from incoherent maritime radar data. IET Radar Son Nav. 5(2), 144-154 (2011). doi:10.1049/iet-rsn.2010.0001 doi:10.1186/1687-6180-2012-84

Cite this article as: Vicen-Bueno et al:: Estimate of significant wave height from non-coherent marine radar images by multilayer perceptrons. EURASIP Journal on Advances in Signal Processing 2012 2012:84.
Submit your manuscript to a SpringerOpen ${ }^{\mathcal{O}}$ journal and benefit from:

- Convenient online submission

- Rigorous peer review

- Immediate publication on acceptance

- Open access: articles freely available online

- High visibility within the field

- Retaining the copyright to your article

Submit your next manuscript at $\gg$ springeropen.com 